\title{
Crack nucleation in saturated porous media
}

\author{
L. Dormieux ${ }^{\dagger}$, E. Lemarchand ${ }^{\dagger}$, T. Carlioz ${ }^{\dagger}$ and L. Jeannin* \\ † Laboratoire Navier, Ecole des Ponts Paristech, 6 et 8, avenue Blaise Pascal 77455 Marne-la-Vallée, France \\ * Storengy, Immeuble Djiin, 12 rue Raoul Nordling, 92274 Bois-Colombes Cedex, France
}

\begin{abstract}
SUMMARY
Crack nucleation has been the subject of important contributions in the two last decades. However, it seems that few attention has been granted to the case of saturated porous media. This is the question adressed in the present paper which is devoted to nucleation in traction mode. From a physical point of view, nucleation is a sudden phenomenon, so that the material response is both adiabatic and undrained. In the spirit of the variational approach, the nucleated crack is viewed as the final state of a region of space in which the material undergoes a full damage process. In traction mode, the opening of a saturated crack in undrained condition induces a drop of fluid pressure. In case of low fluid compressibility, the presence of the fluid delays the brittle failure usually associated with nucleation, as long as the fluid pressure remains above the saturation vapour pressure. Nucleation is therefore possible only if a partial vaporization of the fluid takes place. Copyright (c) 0000 John Wiley \& Sons, Ltd.
\end{abstract}

Received ...

KEY WORDS: crack nucleation, saturated porous media, stability analysis, damage model, saturation vapour pressure

\section{INTRODUCTION}

Since the early works of Griffith [1], the presence of cracks in mechanical structures has been recognized as a scientific challenge of paramount importance. It has focused the attention of very numerous researchers. The fundamental aspects of the question, including strain and stress singularities and propagation are now presented in many reference papers and books (e. g. [2], [8], [9]). The prediction of the effect of microcracks on the linear elastic stiffness was also addressed in the context of length scale transition techniques based on various mathematical crack models. This includes, on one hand, the Griffith crack model in which the crack is a line in 2D or a surface in 3D (e.g. [10]) and, on the other hand, the elliptic or ellipsoidal crack models (e.g. [11], [12]). These two model types yield consistent results as regards the prediction of effective properties of cracked materials (see [3]). This result is extended to the rate of energy release at section 3.2. More recently, the issue of the nucleation of cracks has received important contributions. By definition, the nucleation is the transition from the sound material to the cracked material, when a critical loading level is reached. By nature, it can be viewed as the propagation of a crack, starting from a length equal to 0 , but in a very short period of time. In the variational approach (e.g. [5], [6]), the crack is viewed as the final state of some region of the structure in which the material has reached a full damage level. The determination of the loading required for the crack nucleation and of the geometry of the nucleated crack is achieved by a double variational principle concerning the displacement on the one hand and the damage on the other hand. The energy function to which this variational procedure is applied comprises a term accounting for damage gradient in the medium. This approach requires by nature to follow the evolution of the system in a step-by-step procedure. In contrast, the double criterion first proposed in [14] does not go through the time process leading to nucleation. The emphasis is put on the fact that the propagation that produces the crack is not stable in the sense that the crack length increases under constant loading as long as it has not reached the nucleation length, if the latter exists. Therefore, the idea is to deal with nucleation as a discontinuity 
with respect to time in the state of the system. It relies upon an energy balance between the initiation of the nucleation and its end which includes a variation of the potential energy $\delta E_{p}$, of the kinetic energy $\delta K$ and a dissipation $G_{c} \delta S$ proportional to the created crack area $\delta S$ ( $G_{c}$ is the fracture energy). Observing that the increment of the kinetic energy is necessarily a positive number if the initial state is at equilibrium, a very simple necessary condition of nucleation is derived in the form of an energy criterion:

$$
R_{\text {inc }}=-\frac{\delta E_{p}}{\delta S} \geq G_{c}
$$

Although the expression in the lefthand side evokes the classical differential rate of energy release

$$
R_{\mathrm{diff}}=-\partial E_{p} / \partial S
$$

which appears in the issue of crack propagation, it is important to notice that the quantity at stake here is an incremental rate and not a derivative. In [14], it is suggested that (1) must be combined with a stress criterion to be fulfilled in the area where the crack is nucleated in order to determine the critical loading level of nucleation as well as its extent. [15] recently proposed an alternative to this stress criterion. Observing that the nucleation of a stable crack requires, by nature, that the loading level does not fulfill the condition of propagation of the nucleated crack, it is argued that the geometry of the nucleated crack should correspond to a maximum of the incremental rate of energy release viewed as a function of a characteristic geometrical crack parameter (typically the length in plane strains, see figure 1). The critical loading level is then determined from the condition that this maximum is equal to $G_{c}$, replacing in (1) the inequality by an equality. In figure 1, the blue curve is above the red one as long as $\ell<\ell^{*}$, meaning that the condition of propagation is fullfilled: no stable crack can be observed. In contrast, if $\ell>\ell^{*}$, the blue curve is below the red one, meaning that no propagation can take place.

The variational approach as well as the energy criterion have been illustrated and provided valuable results in a number of applications. Nevertheless, the issue of nucleation clearly remains a subject of intensive research. As regards the possible implementation of these approaches in the case of multiphase geomaterials, the poromechanical coupling appears as the first issue to be considered. More explicitely, the question is the role of the fluid in the nucleation process. [15] argued that nucleation being by nature a sudden phenomenon, the process should be considered in adiabatic (no heat flow) and undrained (no fluid mass flow) conditions. In the framework of thermoporoelasticity, this implies to characterize the constitutive behavior of the medium in which the nucleated crack is embedded by an adiabatic undrained stiffness tensor, so that formally a linear relationship between (total) stress and strain is retrieved. However, the question of the role of the fluid is by far not fully answered: it remains to clarify the essential issue of the boundary conditions which have to be considered on the lips of the nucleated crack.

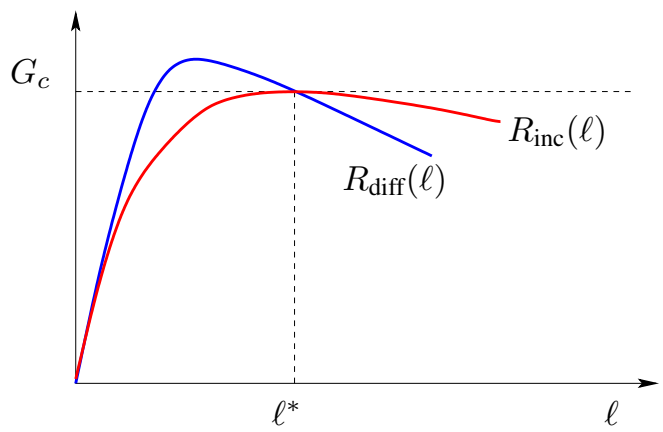

Figure 1. Characterization of the nucleated crack length $\ell^{*}$ : differential and incremental rates of energy release.

At first sight, a straightforward extension for the nucleation of a Griffith crack in an undrained medium could be to prescribe an undrained behavior of the constitutive material while keeping the same kind of boundary conditions on the crack lips, as for a non porous medium. For instance, in traction mode, the 
lips of the nucleated crack would be free of stress. Clearly enough, if this is adequate, the nucleation of crack in saturated porous materials is not an issue at all, in so far as it strictly amounts to nucleation in non porous media, with one exception, namely the use of the undrained stiffness tensor instead of the drained one. In fact, such an extension is a bit hasty: The model confuses the physical nature of a crack with its mathematical model as a surface (or a line in plane strains) which, by nature, has no volume and as such, does not contain any material phase (neither solid nor fluid). In contrast, if the crack can be viewed as a material system in full damage state, as highlighted in the damage gradient models, then the solid phase has lost its stiffness but the presence of a fluid implies that a pressure is a priori acting at the boundary of the crack, i.e. on the crack lips. The key question as regards the determination of the rate of energy release is the value of this fluid pressure. This is, in short, the subject addressed in the present paper.

Section 2 briefly presents the thermodynamics of nucleation in adiabatic and undrained conditions. In the line of [14] and [15], a criterion of nucleation based on the incremental rate of energy release is proposed. In order to provide a reference in view of forthcoming comparison with the saturated porous case, the non porous case is discussed first at section 3. Section 4 focuses on the poromechanical coupling.

\section{CRITERION FOR NUCLEATION}

Let $\Omega$ denote the mechanical system in which possible crack nucleation is considered. The geometry of the crack is described by a formal parameter $\ell$. This is for instance the length in $2 \mathrm{D}$ or the area in $3 \mathrm{D}$. It controls the energy $G_{c} \ell$ that is dissipated during nucleation.

The created crack $\mathcal{C}(\ell)$ is viewed as a material system since it is the residual state of a subdomain of $\Omega$ in which the material undergoes a full damage process at the end of which it has lost its stiffness. As already mentioned, this description is also underlying the variational approach (e.g. [5]). As compared to its length, $\mathcal{C}(\ell)$ possesses some non zero thickness, though the latter is infinitesimal. It is therefore relevant to define its thermodynamical and mechanical variables and functions, such as strain, stress, stiffness tensors and internal energy. From a mathematical point of view, the 2-dimensional description of $\mathcal{C}(\ell)$ by a surface in 3D (resp. 1-dimensional in 2D) is of course the most convenient. It should however be kept in mind that this is only a mathematical idealization. For instance, nucleation viewed as a full damage process removes the elastic stiffness of the solid inside $\mathcal{C}(\ell)$ but not the heat capacity, so that the material keeps its ability to store internal energy. Besides, the fluid phase in $\mathcal{C}(\ell)$ has a finite compressibility. In undrained conditions, it therefore contributes to the spherical part of the undrained stiffness tensor, and this contribution is not subjected to damage.

Let $\Omega(\ell)=\Omega \backslash \mathcal{C}(\ell)$ denote the complementary of the crack in $\Omega$. By definition, as long as $\dot{\ell}=0, \Omega(\ell)$ undergoes reversible evolutions only.

Crack nucleation is regarded as a sudden phenomenon as compared to the characteristic times of fluid and heat diffusion (see e.g. [16]). Fluid and heat flow are therefore neglected at the time scale of nucleation. Apart from this point, the thermodynamics of nucleation follows a standard reasoning recalled hereafter (see e.g. [3],[8]) and nucleation could be defined as an adiabatic undrained crack propagation from 0 to $\ell$. However, adiabatic conditions lead to select the internal energy rather than the free energy for the formulation of the thermodynamics principles.

The starting point is the first principle: In adiabatic conditions, it states that the mechanical energy provided to the system $\Omega$ is transformed into internal energy $\mathcal{U}$ and kinetic energy $K$ :

$$
\dot{\mathcal{U}}+\dot{K}=\mathcal{P}_{e}
$$

For simplicity, we assume that the mechanical loading $\mathcal{L}$ is defined by body forces $\boldsymbol{F}$, prescribed surface forces $\lambda_{T}(t) \boldsymbol{T}^{0}$ on $\partial \Omega_{T}$, prescribed displacements $\lambda_{\xi}(t) \boldsymbol{\xi}^{0}$ on $\partial \Omega_{\xi}$, where $\lambda_{T}(t)$ and $\lambda_{\xi}(t)$ are given scalar time functions while the boundary of the material system is $\partial \Omega=\partial \Omega_{\xi} \cup \partial \Omega_{T}$ :

$$
\mathcal{P}_{e}=\int_{\Omega} \boldsymbol{F} \cdot \dot{\boldsymbol{\xi}} d V+\lambda_{T}(t) \int_{\partial \Omega_{T}} \boldsymbol{T}^{0} \cdot \dot{\boldsymbol{\xi}} d S+\dot{\lambda}_{\xi}(t) \int_{\partial \Omega_{\xi}} \boldsymbol{T} \cdot \boldsymbol{\xi}^{0} d S
$$


The velocity is the sum of the contribution of the crack nucleation (under steady loading conditions) and that of the loading increment $\dot{\boldsymbol{\xi}}_{\mid \ell}$ (under constant crack length):

$$
\dot{\boldsymbol{\xi}}=\frac{\partial \boldsymbol{\xi}}{\partial \ell} \dot{\ell}_{\mathcal{L}}+\dot{\boldsymbol{\xi}}_{\mid \ell}
$$

Accordingly, the internal (resp. kinetic) energy is the sum of two terms, namely $(\partial \mathcal{U} / \partial \ell)_{\mid \mathcal{L}} \dot{\ell}$ (resp. $(\partial K / \partial \ell)_{\mid \mathcal{L}} \dot{\ell}$ and $\dot{\mathcal{U}}_{\mid \ell}\left(\right.$ resp. $\left.\dot{K}_{\mid \ell}\right)$, which correspond to the contributions of crack nucleation and of the loading increment:

$$
\dot{\mathcal{U}}=\frac{\partial \mathcal{U}}{\partial \ell}{ }_{\mid \mathcal{L}} \dot{\ell}+\dot{\mathcal{U}}_{\mid \ell} \quad ; \quad \dot{K}=\frac{\partial K}{\partial \ell}{ }_{\mid \mathcal{L}} \dot{\ell}+\dot{K}_{\mid \ell}
$$

Similarly, the mechanical work is splitted into two terms $\mathcal{P}_{e \mid \mathcal{L}}$ and $\mathcal{P}_{e \mid \ell}$. Under steady loading conditions, the mechanical power is due to crack propagation only:

$$
\mathcal{P}_{e \mid \mathcal{L}}=\frac{\partial \Phi}{\partial \ell} \mid \mathcal{L} \quad \dot{\ell} \quad \text { with } \quad \Phi=\int_{\Omega} \boldsymbol{F} \cdot \boldsymbol{\xi} d V+\lambda_{T}(t) \int_{\partial \Omega_{T}} \boldsymbol{T}^{0} \cdot \boldsymbol{\xi} d S
$$

where $\Phi$ is the potential of the given loads. In turn, at constant crack length, the standard principle of virtual work readily yields:

$$
\mathcal{P}_{e \mid \ell}=\dot{K}_{\mid \ell}+\int_{\Omega} \boldsymbol{\sigma}: \dot{\varepsilon}_{\mid \ell} d V
$$

where $\sigma$ and $\varepsilon$ denote the local stress and strain fields. Let $u$ denote the volume density of internal energy. By nature, it is a function of the strain and of the entropy density $s$. However, at constant crack length, the evolution being reversible and adiabatic, it is also isentropic. The strain work $\sigma: \dot{\varepsilon}_{\mid \ell}$ is therefore equal to $\dot{u}_{\mid \ell}$ and the corresponding mechanical work reads:

$$
\mathcal{P}_{e \mid \ell}=\dot{K}_{\mid \ell}+\dot{\mathcal{U}}_{\mid \ell}
$$

Eventually, the combination of (2) and (3) yields:

$$
\frac{\partial}{\partial \ell} \mid \mathcal{L}(\mathcal{U}-\Phi+K)=0
$$

To this stage, it is important to observe that $\mathcal{U}$ is the total internal energy of the material system. Let $U$ and $U_{\mathcal{C}}$ respectively denote the internal energy of $\Omega(\ell)$ and of $\mathcal{C}(\ell)$. As an extensive quantity, the total internal energy $\mathcal{U}$ is the sum $U+U_{\mathcal{C}}$. (5) now takes the form:

$$
-\frac{\partial}{\partial \ell_{\mid \mathcal{L}}}(U-\Phi+K)={\frac{\partial U_{\mathcal{C}}}{\partial \ell}}_{\mid \mathcal{L}}
$$

It remains to integrate the above equation w.r.t. $\ell$, under the constant loading $\mathcal{L}$, over the process of nucleation. Let $U_{p}$ denote the potential energy $U-\Phi$. recalling that the initial state is an equilibrium (no kinetic energy), one obtains:

$$
U_{p}(0)-U_{p}(\ell)=U^{F D}+K(\ell)
$$

where $U^{F D}=U_{\mathcal{C}}(\ell)-U_{\mathcal{C}}(0)$ is the energy that was necessary for the nucleation. Due to adiabatic and undrained conditions, it is stored in the crack at the end of nucleation. This highlights the difference between the free and internal energies: the nucleated crack has no free energy but stores in adiabatic conditions the heat dissipated by the full damage process. (7) appears as the adiabatic counterpart of the isothermal energy balance derived in [14]. It states that the energy release of the bulk is the sum of the energy dissipated in the nucleation and of kinetic energy. The latter being a positive quantity, a necessary condition for nucleation reads:

$$
U_{p}(0)-U_{p}(\ell) \geq U^{F D}
$$

If we assume that the energy necessary for nucleation is proportional to the created crack surface $\ell$ $\left(U^{F D}=G_{c} \ell\right)$, the criterion for nucleation takes the form:

$$
\frac{U_{p}(0)-U_{p}(\ell)}{\ell} \geq G_{c}
$$

Again, the above criterion and that derived in [14] look very much alike (see (1)), the only difference being that the internal energy has replaced the free energy. In the use of (8), one should keep in mind its status of necessary condition for the nucleation of a crack with surface (resp. length) $\ell$. 


\section{APPLICATION IN THE NON POROUS CASE}

\section{1. energy release rate for a spheroidal (resp. elliptic) crack}

For the sake of simplicity and for comparative purposes, let us start with the case of a linear elastic non porous material. The isothermal and adiabatic stiffness tensors are $\mathbb{C}_{0}^{\theta}$ and $\mathbb{C}_{0}^{a d}$ respectively. In this section, we consider the possibility of the nucleation of a flat ellipsoidal (resp. elliptic in 2D) crack $\mathcal{E}$. This means that we examine a damage process at the end of which the material within the subdomain $\mathcal{E}$ is fully damaged. Introducing a formal damage parameter $d$, the stiffness tensor $\mathbb{C}^{a d}(d)$ therefore starts from $\mathbb{C}_{0}^{a d}$ and ends up with 0 . Accordingly, the stress state in $\mathcal{E}$ at the end of this process is 0 . We shall prove at section 3.2 that this crack model is entirely consistent with the prediction obtained with the Griffith crack model.

The nucleation takes place in an initial state under prescribed asymptotic boundary conditions on the displacement field $\boldsymbol{\xi}$. The natural state (stress equal to 0 , uniform temperature) is taken as reference state:

$$
\xi \underset{|z| \rightarrow \infty}{\sim} E \cdot z
$$

where $\boldsymbol{E}$ is a given symmetric strain tensor. It is assumed that the temperature is uniform and the same as in the reference state. Accordingly, the initial stress state (i.e. prior to nucleation under uniform strain $\boldsymbol{E}$ ) is:

$$
\boldsymbol{\sigma}_{0}=\mathbb{C}_{0}^{\theta}: \boldsymbol{E}
$$

According to section 2, the energy release in this process is the relevant quantity. The elastic energy is not bounded since the medium is infinite. This difficulty is avoided by considering the internal energy $U_{R}$ stored in the subdomain $\mathcal{B}_{R}$ defined by $|\boldsymbol{z}| \leq R$ and $\boldsymbol{z} \notin \mathcal{E}$, and by letting $R \rightarrow \infty$. Note that the boundary of $\mathcal{B}_{R}$ comprises the sphere (resp. circle in $2 \mathrm{D}$ ) $\mathcal{S}_{R}$ and the boundary $\partial \mathcal{E}$ of $\mathcal{E}$. The energy release rate is then the limit:

$$
\lim _{R \rightarrow \infty} \frac{U_{R}(0)-U_{R}(\ell)}{\ell}
$$

In the above limit, $U_{R}(0)$ is the energy of the domain $\mathcal{B}_{R}$ prior to nucleation and reads :

$$
U_{R}(0)=\frac{\left|\mathcal{B}_{R}\right|}{2} \boldsymbol{E}: \mathbb{C}_{0}^{\theta}: \boldsymbol{E}
$$

where $\left|\mathcal{B}_{R}\right|$ is the measure (volume in $3 \mathrm{D}$ or area in $2 \mathrm{D}$ ) of $\mathcal{B}_{R}$. In turn, $U_{R}(\ell)$ is the internal energy stored in $\mathcal{B}_{R}$ after the nucleation process. In view of its derivation, let $\delta \varepsilon$ (resp. $\delta \boldsymbol{\xi}$ ) denote the perturbation of the strain (resp. of the displacement) induced by nucleation. The total strain $\varepsilon$ is therefore

$$
\varepsilon=\boldsymbol{E}+\delta \varepsilon
$$

The strain perturbation is related to the post-nucleation stress state $\sigma$ by the adiabatic linear elastic stiffness tensor :

$$
\boldsymbol{\sigma}=\boldsymbol{\sigma}_{0}+\mathbb{C}_{0}^{a d}: \delta \varepsilon
$$

We note that $\delta \boldsymbol{\varepsilon} \rightarrow 0$ and $\delta \boldsymbol{\xi} \rightarrow 0$ when $|\boldsymbol{z}| \rightarrow \infty$ since the strain and displacement at infinity are prescribed. The strain field $\delta \varepsilon$ is defined outside $\mathcal{E}$ but can be smoothly extended into $\mathcal{E}$ by the solution $\delta \varepsilon^{\mathcal{E}}$ to the Eshelby problem depicted at figure 2 (see Appendix 7.2). By application of (60) in which $\boldsymbol{E}$ is $0, \boldsymbol{\tau}^{\mathcal{E}}$ is 0 , $\mathbb{C}^{\mathcal{E}}$ is $0, \mathbb{C}$ is $\mathbb{C}_{0}^{a d}$ and $\boldsymbol{\tau}$ is $\boldsymbol{\sigma}_{0}$, it is found that:

$$
\delta \mathcal{E}^{\mathcal{E}}=\left(\mathbb{0}-\mathbb{P}_{0}^{a d}: \mathbb{C}_{0}^{a d}\right)^{-1}: \mathbb{P}_{0}^{a d}: \sigma_{0}
$$

where $\llbracket$ is the fourth order identity tensor and $\mathbb{P}_{0}^{a d}$ is the Hill tensor of $\mathcal{E}$ embedded in the linear elastic medium with stiffness $\mathbb{C}_{0}^{a d}$ and we have (energy change in adiabatic evolution with prestress):

$$
U_{R}(\ell)=U_{R}(0)+\int_{\mathcal{B}_{R}} \sigma_{0}: \delta \varepsilon+\frac{1}{2} \delta \varepsilon: \mathbb{C}_{0}^{a d}: \delta \varepsilon d V
$$




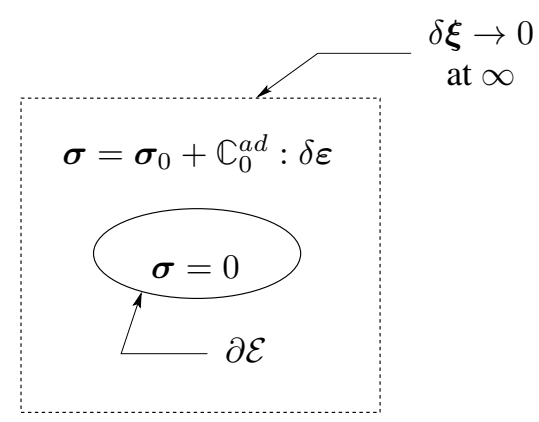

Figure 2. The post-nucleation mechanical problem in adiabatic conditions

which is more conveniently put in the form:

$$
U_{R}(\ell)=U_{R}(0)+\int_{\mathcal{B}_{R}} \frac{1}{2} \delta \varepsilon: \sigma_{0} d V+\int_{\mathcal{B}_{R}} \frac{1}{2} \delta \varepsilon: \sigma d V
$$

We now transform the integrals in (12) into boundary integrals. Observe that the stress vector $\boldsymbol{\sigma} \cdot \boldsymbol{n}$ is 0 on the boundary of $\mathcal{E}$, that is, on the internal boundary of $\mathcal{B}_{R}$. This yields:

$$
\int_{\mathcal{B}_{R}} \frac{1}{2} \delta \varepsilon: \boldsymbol{\sigma} d V=\int_{\mathcal{S}_{R}} \frac{1}{2} \delta \boldsymbol{\xi} \cdot \boldsymbol{\sigma} \cdot \boldsymbol{n} d S
$$

where $\boldsymbol{n}$ is the outwards (w.r.t. $\mathcal{B}_{R}$ ) unit normal at any point on $\mathcal{S}_{R}$. Similarly, we have:

$$
\int_{\mathcal{B}_{R}} \frac{1}{2} \delta \varepsilon: \boldsymbol{\sigma}_{0} d V=-\frac{|\mathcal{E}|}{2} \delta \varepsilon^{\mathcal{E}}: \boldsymbol{\sigma}_{0}+\int_{\mathcal{S}_{R}} \frac{1}{2} \delta \boldsymbol{\xi} \cdot \boldsymbol{\sigma}_{0} \cdot \boldsymbol{n} d S
$$

The fact that $\delta \boldsymbol{\xi} \rightarrow 0$ at infinity suggests that the limit at $R \rightarrow \infty$ of the boundary integrals on $\mathcal{S}_{R}$ is 0 . This point can be readily confirmed in plane strains by means of complex analysis [3]. In this case, it is therefore concluded that the energy release is:

$$
\lim _{R \rightarrow \infty} U_{R}(0)-U_{R}(\ell)=\frac{|\mathcal{E}|}{2} \delta \mathcal{E}^{\mathcal{E}}: \sigma_{0}
$$

In other words, the energy release rate is:

$$
\frac{U(0)-U(\ell)}{\ell}=\frac{|\mathcal{E}|}{2 \ell} \delta \varepsilon^{\mathcal{E}}: \sigma_{0}
$$

The interest of (15) lies in the fact that a simple analytical expression can be derived when the aspect ratio of the spheroidal (resp. elliptic) domain $\mathcal{E}$ tends to 0 , as required in view of a crack model. Let start with the flat spheroidal model, in which $a$ is the radius and $c$ the axial half-length. The aspect ratio is $X=c / a$. In the $3 \mathrm{D}$ case, the geometrical parameter $\ell$ is the area of the crack: $\ell=\pi a^{2}$. The important feature is the existence of the limit (see e.g. [3]):

$$
\mathbb{T}^{a d}=\lim _{X \rightarrow 0} X\left(\mathbb{\square}-\mathbb{P}_{0}^{a d}(X): \mathbb{C}_{0}^{a d}\right)^{-1}
$$

This means that the leading term in the expression (10) of $\delta \varepsilon^{\mathcal{E}}$ is $O(1 / X)$. The tensor $\mathbb{T}^{a d}$ satisfies the minor but not the major symmetry conditions. A simple analytical expression of its coefficients is available for instance in the isotropic case (other coefficients are $0, e_{3}$ denotes the direction normal to the crack plane):

$$
\begin{gathered}
T_{3311}^{a d}=\frac{4 \nu_{0}^{a d}\left(1-\nu_{0}^{a d}\right)}{\pi\left(1-2 \nu_{0}^{a d}\right)} \quad ; \quad T_{3333}^{a d}=\frac{4\left(1-\nu_{0}^{a d}\right)^{2}}{\pi\left(1-2 \nu_{0}^{a d}\right)} \quad ; \quad T_{2323}^{a d}=\frac{2\left(1-\nu_{0}^{a d}\right)}{\pi\left(2-\nu_{0}^{a d}\right)} \\
T_{3322}^{a d}=T_{3311}^{a d} \quad ; \quad T_{1313}^{a d}=T_{2323}^{a d}
\end{gathered}
$$


where $\nu_{0}^{a d}$ is the Poisson coefficient of the material with stiffness tensor $\mathbb{C}_{0}^{a d}$. Introducing $|\mathcal{E}|=4 \pi a^{3} X / 3$ and (10) into (15), the limit of the rate of energy release when $X \rightarrow 0$ reads:

$$
\frac{U(0)-U(\ell)}{\ell}=\frac{2 a}{3} \sigma_{0}: \mathbb{T}^{a d}: \mathbb{P}_{0}^{a d}: \sigma_{0}
$$

For example, consider the isotropic loading case with $\sigma_{0}=\Sigma_{0} \delta$. The above expression reads:

$$
\frac{U(0)-U(\ell)}{\ell}=\frac{2 a}{3 \pi} \frac{\Sigma_{0}^{2}}{\mathcal{M}_{o}}+O(X) \quad \text { where } \quad \mathcal{M}_{o}=\frac{3 k_{0}^{a d}+\mu_{0}^{a d}}{3 k_{0}^{a d}+4 \mu_{0}^{a d}} \mu_{0}^{a d}
$$

In turn, in the plane strain 2D case, the flat elliptic crack is characterized by the half-opening $c$, the halflength $a$ and the aspect ratio $X=c / a$. The definition of tensor $\mathbb{T}^{a d}$ is identical. In the isotropic case, the non-zero coefficients are ( $e_{2}$ denotes the direction normal to the crack plane):

$$
T_{2211}^{a d}=\frac{2 \nu_{0}^{a d}\left(1-\nu_{0}^{a d}\right)}{1-2 \nu_{0}^{a d}} \quad ; \quad T_{2222}^{a d}=\frac{2\left(1-\nu_{0}^{a d}\right)^{2}}{1-2 \nu_{0}^{a d}} \quad ; \quad T_{1212}^{a d}=\frac{1-\nu_{0}^{a d}}{2}
$$

Now, the geometrical parameter $\ell$ is the large axis length $2 a$ and $|\mathcal{E}|=\pi a^{2} X$. It is found that:

$$
\frac{U(0)-U(\ell)}{\ell}=\frac{\pi a}{4} \sigma_{0}: \mathbb{T}^{a d}: \mathbb{P}_{0}^{a d}: \sigma_{0}
$$

From a mathematical point of view, the fact that the limit when $X \rightarrow 0$ of the rate of energy release is not 0 (while $|\mathcal{E}| \rightarrow 0$ ) is intrinsically related to the property $\delta \varepsilon^{\mathcal{E}}=O(1 / X)$.

If the difference between the isothermal and adiabatic stiffness is disregarded, the superscript $a d$ can be omitted and the leading term in the quantity $\sigma_{0}: \mathbb{T}: \mathbb{P}_{0}^{a d}: \sigma_{0}$ in (18) and (21) also reads:

$$
\sigma_{0}: \mathbb{T}: \mathbb{P}_{0}: \sigma_{0}=\boldsymbol{E}: \mathbb{C}_{0}: \mathbb{T}: \boldsymbol{E}
$$

This approximation is made in the two next sections 3.2 and 3.3 .

\subsection{Comparison with the Griffith crack model}

Let consider plane strain conditions. The purpose of this section is to derive the energy release in the nucleation of a rectilinear Griffith crack of length $\ell$ in mode I from Irwin's formula:

$$
-\frac{\partial U}{\partial \ell}=\frac{K_{I}^{2}}{Y_{0}}\left(1-\nu_{0}^{2}\right)
$$

where $K_{I}$ is the stress intensity factor and $Y_{0}$ the Young's modulus. Clearly enough, the geometrical model of the crack and the methodology are now completely different from the concept presented at section 3.1. The aim is to retrieve the result (21) in order to show the consistency of the elliptic crack model with the classical Griffith crack model as regards the prediction of nucleation.

To do so, an asymptotic stress state $\sigma^{\infty}$ compatible with plane strain conditions is applied at infinity:

$$
\boldsymbol{\sigma}^{\infty}=\sigma_{\infty}\left(\boldsymbol{e}_{1} \otimes \boldsymbol{e}_{1}+\boldsymbol{e}_{2} \otimes \boldsymbol{e}_{2}+2 \nu_{0} \boldsymbol{e}_{3} \otimes \boldsymbol{e}_{3}\right)
$$

The associated strain at infinity is

$$
\boldsymbol{E}=\left(1+\nu_{0}\right)\left(1-2 \nu_{0}\right) \frac{\sigma_{\infty}}{Y_{0}}\left(\boldsymbol{e}_{1} \otimes \boldsymbol{e}_{1}+\boldsymbol{e}_{2} \otimes \boldsymbol{e}_{2}\right)
$$

On the one hand, if the previous elliptic crack model is considered, the energy release rate is derived from (21) in which (20) and (23) are introduced:

$$
\frac{U(0)-U(\ell)}{\ell}=\frac{\pi \ell \sigma_{\infty}^{2}}{4 Y_{0}}\left(1-\nu_{0}^{2}\right)
$$


On the other hand, let us consider the Griffith crack model and a rectilinear crack of length $\ell$ and normal $e_{3}$. The stress intensity factor $K_{I}$ is $\sigma_{\infty} \sqrt{\pi \ell / 2}$, so that (22) yields:

$$
-\frac{\partial U}{\partial \ell}=\frac{\sigma_{\infty}^{2} \pi \ell}{2 Y_{0}}\left(1-\nu_{0}^{2}\right)
$$

It is readily seen that (24) is retrieved by integration of the above equation w.r.t. $\ell$. This confirms the consistency of the usual crack description (Griffith model) with a concept of full damage within a flat elliptic domain. As far as energy balance is concerned, this result validates the description of nucleation as the brutal transition from a sound to a fully damage state in a thin region of space which is going to become the crack. It also motivates the extension of such an approach of nucleation to saturated porous media.

\subsection{Stability analysis}

\subsubsection{Constitutive equations of damage theory}

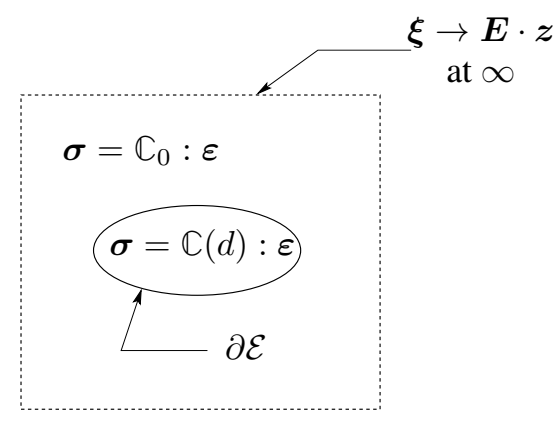

Figure 3. Damage process under prescribed asymptotic displacements

As a possible alternative to the reasoning based on the rate of energy release (15), we examine in this section the possibility to simulate the damage process in $\mathcal{E}$ leading to the crack (see figure 3 ). To do so, one first needs a damage model providing the stiffness tensor as a function $\mathbb{C}(d)$ decreasing from $\mathbb{C}_{0}$ to 0 , as the damage parameter $d$ increases (for instance) from 0 to 1 . Then, the damage criterion is written as a bound on the thermodynamic force associated with damage (e.g. [3],[7]):

$$
\dot{d}\left\{\begin{array}{l}
=0 \text { if }-\frac{1}{2} \varepsilon: \mathbb{C}^{\prime}(d): \varepsilon<g_{c} \\
\geq 0 \text { if }-\frac{1}{2} \varepsilon: \mathbb{C}^{\prime}(d): \varepsilon=g_{c}
\end{array}\right.
$$

in which $g_{c}$ is the critical energy density. Thus, the damage criterion provides a link between the local strain and the corresponding damage parameter. Finally, the time derivative of this damage criterion relates the strain and damage rates :

$$
\left(\frac{1}{2} \varepsilon: \mathbb{C}^{\prime \prime}(d): \varepsilon\right) \dot{d}+\dot{\varepsilon}: \mathbb{C}^{\prime}(d): \varepsilon=0
$$

Introducing (26) into the time derivative of the constitutive equation $\sigma=\mathbb{C}(d): \varepsilon$ provides the expression of the tangent stiffness tensor which relates the stress and strain rates: $\dot{\sigma}=\mathbb{C}_{t}: \dot{\varepsilon}$. It depends on the damage rate $\dot{d}$ :

$$
\begin{aligned}
\dot{d}>0 \Rightarrow \mathbb{C}_{t} & =\mathbb{C}(d)-2 \frac{\left(\mathbb{C}^{\prime}(d): \varepsilon\right) \otimes\left(\mathbb{C}^{\prime}(d): \varepsilon\right)}{\varepsilon: \mathbb{C}^{\prime \prime}(d): \varepsilon} \\
\dot{d} & =0 \Rightarrow \mathbb{C}_{t}=\mathbb{C}(d)
\end{aligned}
$$

In the present case, uniform damage is assumed within the domain $\mathcal{E}$, while $d=0$ outside of $\mathcal{E}\left(\mathbb{C}(0)=\mathbb{C}_{0}\right)$. The question is twofold: we have to relate the damage parameter to the loading history $\boldsymbol{E}(t)$ and to discuss the stability of the response of the system. In fact, we shall see in the next section that the stability condition is never achieved in the non porous case. This is consistent with a brittle failure at a critical loading level usually associated with the concept of nucleation. In contrast, the saturated case will exhibit a different behavior. 


\subsubsection{Application of Hill's criterion}

The displacement, strain and damage fields being given everywhere in the structure, the question is to determine whether this state of the structure is a stable equilibrium or not. Hill's criterion of stability was originally introduced in the framework of plasticity ([17]). This original formulation is readily adapted to the framework of damage ([15]) and yields a formally identical criterion of stability. In the present problem, the idea is to consider a virtual perturbation $\widehat{\boldsymbol{\delta} \xi}$ of the displacement field, which vanishes at infinity (kin. adm. with 0 ). If this perturbation could take place, it would be associated with a necessarily positive increment of kinetic energy. This would imply

$$
\int_{\Omega} \widehat{\boldsymbol{\delta \sigma}}: \widehat{\delta \varepsilon} d V \leq 0
$$

Conversely, in order to ensure that the considered state is a stable equilibrium, a sufficient condition is that

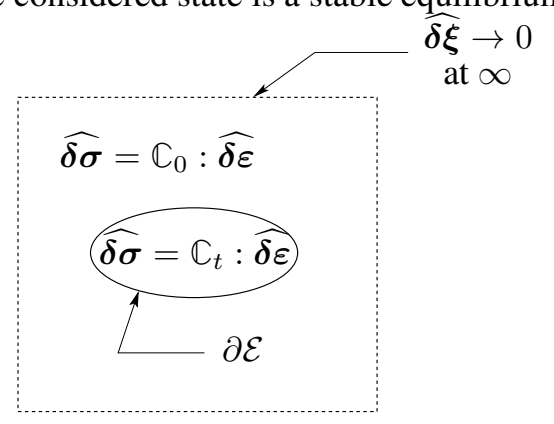

Figure 4. Perturbation of the current state

the above inequality is never satisfied. In other words, Hill's stability criterion thus reads:

$$
(\forall \widehat{\boldsymbol{\delta} \xi} \text { k.a. } 0) \quad \int_{\Omega} \widehat{\boldsymbol{\delta} \sigma}: \widehat{\boldsymbol{\delta} \varepsilon} d V>0
$$

In (29), $\widehat{\delta \sigma}$ and $\widehat{\delta \varepsilon}$ are related by the state equation:

$$
\widehat{\delta \sigma}=\mathbb{C}_{t}: \widehat{\delta \varepsilon}
$$

where the expression of $\mathbb{C}_{t}$ is given in (27) and (28), depending on the damage rate. The criterion requires to consider all relevant perturbation fields. In practice, it seems reasonable to assume a uniform perturbation strain $\widehat{\delta \varepsilon}_{\mathcal{E}}$ inside the subdomain $\mathcal{E}$. Up to a rigid body motion, the corresponding displacement is $\widehat{\delta \xi}=\widehat{\delta \varepsilon} \mathcal{E} \cdot z$. Outside of $\mathcal{E}$, any smooth extension of the displacement meeting the boundary condition $\widehat{\delta \xi}=\widehat{\boldsymbol{\delta}}_{\mathcal{E}} \cdot \boldsymbol{z}$ on $\partial \mathcal{E}$ and vanishing at infinity (i.e. kin. adm. with $\widehat{\boldsymbol{\delta}}_{\mathcal{E}}$ ) must be considered (see figure 4). (29) now reads:

$$
(\forall \widehat{\boldsymbol{\delta} \varepsilon} \mathcal{E} \neq 0)\left(\forall \widehat{\boldsymbol{\delta} \xi} k . a . \widehat{\boldsymbol{\delta}}_{\mathcal{E}}\right) \quad \int_{\Omega \backslash \mathcal{E}} \widehat{\boldsymbol{\delta} \varepsilon}: \mathbb{C}_{0}: \widehat{\boldsymbol{\delta} \varepsilon} d V+|\mathcal{E}| \widehat{\boldsymbol{\delta}}_{\mathcal{E}}: \mathbb{C}_{t}: \widehat{\boldsymbol{\delta}}_{\mathcal{E}}>0
$$

At this stage, it is instructive to consider the solution $\boldsymbol{\xi}^{e l}$ (resp. $\epsilon^{e l}$ ) of the auxiliary problem of elasticity (see figure 5) defined on $\Omega \backslash \mathcal{E}$ by the uniform stiffness tensor $\mathbb{C}_{0}$ and the boundary conditions $\boldsymbol{\xi}^{e l} \rightarrow 0$ at infinity and $\boldsymbol{\xi}^{e l}=\widehat{\boldsymbol{\delta}}_{\mathcal{E}} \cdot \boldsymbol{z}$ on $\partial \mathcal{E}$. The principle of minimum potential energy states that:

$$
\left(\forall \widehat{\boldsymbol{\delta}} \varepsilon_{\mathcal{E}} \neq 0\right)\left(\forall \widehat{\boldsymbol{\delta} \boldsymbol{\xi}} k . a . \widehat{\boldsymbol{\delta} \varepsilon_{\mathcal{E}}}\right) \quad \int_{\Omega \backslash \mathcal{E}} \widehat{\boldsymbol{\delta} \varepsilon}: \mathbb{C}_{0}: \widehat{\boldsymbol{\delta} \varepsilon} d V \geq \int_{\Omega \backslash \mathcal{E}} \boldsymbol{\epsilon}^{e l}: \mathbb{C}_{0}: \boldsymbol{\epsilon}^{e l} d V
$$

The uniform stress $\widehat{\delta \sigma}_{\mathcal{E}}$ acting on $\partial \mathcal{E}$ that induces the strain $\widehat{\boldsymbol{\delta}}_{\mathcal{E}}$ in the auxiliary problem is again derived from (60):

$$
\widehat{\boldsymbol{\delta}}_{\mathcal{E}}=-\left(\mathbb{\square}-\mathbb{P}_{0}: \mathbb{C}_{0}\right)^{-1}: \mathbb{P}_{0}: \widehat{\boldsymbol{\delta}}_{\mathcal{E}}
$$

The principle of virtual work readily shows that:

$$
\int_{\Omega \backslash \mathcal{E}} \boldsymbol{\epsilon}^{e l}: \mathbb{C}_{0}: \boldsymbol{\epsilon}^{e l} d V=\int_{\partial \mathcal{E}} \boldsymbol{\xi}^{e l} \cdot \widehat{\boldsymbol{\delta}}_{\mathcal{E}} \cdot \boldsymbol{n} d S=-|\mathcal{E}| \widehat{\boldsymbol{\delta}}_{\mathcal{E}}: \widehat{\boldsymbol{\delta}}_{\mathcal{E}}
$$




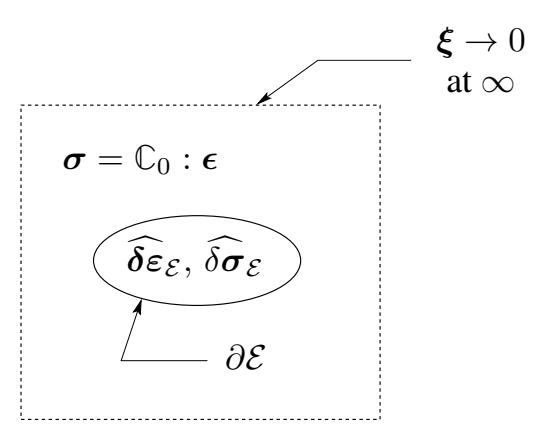

Figure 5. An auxiliary elasticity problem: elastic solution induced in $\Omega \backslash \mathcal{E}$ by uniform strain $\widehat{\boldsymbol{\delta \varepsilon}}_{\mathcal{E}}$ or stress $\widehat{\delta \boldsymbol{\sigma}}_{\mathcal{E}}$ inside $\mathcal{E}$

Combining (31) and (32) with the above result yields:

$$
\left(\forall \widehat{\boldsymbol{\delta}}_{\mathcal{E}} \neq 0\right)\left(\forall \widehat{\boldsymbol{\delta} \xi} k . a . \widehat{\boldsymbol{\delta}}_{\mathcal{E}}\right) \quad \int_{\Omega \backslash \mathcal{E}} \widehat{\boldsymbol{\delta} \varepsilon}: \mathbb{C}_{0}: \widehat{\boldsymbol{\delta} \varepsilon} d V \geq|\mathcal{E}| \widehat{\boldsymbol{\delta}}_{\mathcal{E}}:\left(\mathbb{P}_{0}^{-1}-\mathbb{C}_{0}\right): \widehat{\boldsymbol{\delta}}_{\mathcal{E}}
$$

Eventually, one comes up with the following lower bound:

$\left(\forall \widehat{\boldsymbol{\delta}}_{\mathcal{E}} \neq 0\right)\left(\forall \widehat{\boldsymbol{\delta} \boldsymbol{\xi}} k . a . \widehat{\boldsymbol{\delta}}_{\mathcal{E}}\right) \quad \int_{\Omega \backslash \mathcal{E}} \widehat{\boldsymbol{\delta} \varepsilon}: \mathbb{C}_{0}: \widehat{\boldsymbol{\delta} \varepsilon} d V+|\mathcal{E}| \widehat{\boldsymbol{\delta}}_{\mathcal{E}}: \mathbb{C}_{t}: \widehat{\boldsymbol{\delta}}_{\mathcal{E}} \geq|\mathcal{E}| \widehat{\boldsymbol{\delta}}_{\mathcal{E}}:\left(\mathbb{P}_{0}^{-1}-\mathbb{C}_{0}+\mathbb{C}_{t}\right): \widehat{\boldsymbol{\delta} \varepsilon_{\mathcal{E}}}$

the equality in (33) being reached for $\widehat{\boldsymbol{\delta} \boldsymbol{\xi}}=\boldsymbol{\xi}^{e l}$. Comparing to (30), it is concluded that Hill's criterion reduces to the positive definiteness of the tensor $\mathbb{P}_{0}^{-1}-\mathbb{C}_{0}+\mathbb{C}_{t}$ :

$$
\left(\forall \widehat{\boldsymbol{\delta}}_{\mathcal{E}} \neq 0\right) \quad \widehat{\boldsymbol{\delta}}_{\mathcal{E}}:\left(\mathbb{P}_{0}^{-1}-\mathbb{C}_{0}+\mathbb{C}_{t}\right): \widehat{\boldsymbol{\delta}}_{\mathcal{E}}>0
$$

This conclusion can be made explicit in the case of an isotropic solid phase. We herein consider a class of isotropic damage models in which $\mathbb{C}(d)=3 k(d) \mathbb{J}+2 \mu(d) \mathbb{K}$ with:

$$
\frac{k(d)}{k_{0}}=\frac{\mu(d)}{\mu_{0}}=f(d) \quad \text { with } \quad f(d)=\frac{1-Q d}{1+Q^{\prime} d}
$$

with $Q, Q^{\prime}>0$. Considering the Voigt matrix of $\mathbb{P}_{0}^{-1}-\mathbb{C}_{0}+\mathbb{C}_{t}$ (see section 7.1), a negative eigenvalue associated with a strain mechanism of mode I is readily identified:

$$
\lambda_{I}=-\left(k_{0}+\frac{4}{3} \mu_{0}\right) \frac{Q}{Q^{\prime}} \quad \text { with eigenvector } \quad \widehat{\boldsymbol{\delta} \varepsilon}=\boldsymbol{e}_{3} \otimes \boldsymbol{e}_{3}
$$

In the non porous case, this proves that mode I nucleation of crack cannot be modelled by a stable damage process in a flat spheroid. We shall see that a different conclusion can be drawn in the saturated porous case.

\section{THE SATURATED POROUS MEDIUM}

For the sake of brevity and without loss of generality, we now consider the three-dimensional case only.

\subsection{A first expression of the rate of energy release}

Let us now adapt the developments of section 3.1 to the saturated case. The natural state (pore pressure and stress field equal to 0 ) is taken as a reference. In the initial state prior nucleation, the strain field $\varepsilon$, the pore pressure and the temperature (same as in the reference state) are uniform, respectively equal to $\boldsymbol{E}, p_{0}$ and $T_{0}$. The initial stress field is uniform as well:

$$
\boldsymbol{\sigma}_{0}=\mathbb{C}_{0}^{d, \theta}: \boldsymbol{E}-p_{0} \boldsymbol{B}_{0}^{\theta}
$$


in which $\mathbb{C}_{0}^{d, \theta}$ (resp. $\boldsymbol{B}_{0}^{\theta}$ ) is the stiffness tensor in both drained and isothermal conditions (resp. the Biot tensor in isothermal conditions). In turn, the strain perturbation is related to the post-nucleation stress state $\sigma$ by the adiabatic undrained linear elastic stiffness tensor $\mathbb{C}_{0}^{u, a d}$ (see section 7.3.2):

$$
\boldsymbol{\sigma}=\boldsymbol{\sigma}_{0}+\mathbb{C}_{0}^{u, a d}: \delta \varepsilon
$$

Note that the (undrained) nucleation induces a pore pressure change everywhere. In particular, the pore pressure inside $\mathcal{E}$ varies from the initial value $p_{0}$ to an a priori unknown value $p^{\mathcal{E}}$. The expression of the internal energy (including the contribution of both the solid and fluid phases) is identical to (11) except for the fact that $\mathbb{C}_{0}^{a d}$ is replaced by $\mathbb{C}_{0}^{u, a d}$ (see (70)). Therefore, (12) is not modified. But the great difference lies in the fact that the stress vector $\boldsymbol{\sigma} \cdot \boldsymbol{n}$ on the internal boundary of $\mathcal{B}_{R}$ (which is $\partial \mathcal{E}$ ) is not 0 but is equal to $-p^{\mathcal{E}} \boldsymbol{n}$. Indeed, since the fully damaged material that remains in the post-nucleation state has no stiffness (by definition), the stress state inside $\mathcal{E}$ is the spherical stress tensor $-p^{\mathcal{E}} \boldsymbol{\delta}$. This first impacts the expression of the rate of energy release: While (14) is still valid, (13) is now replaced by:

$$
\int_{\mathcal{B}_{R}} \frac{1}{2} \delta \varepsilon: \boldsymbol{\sigma} d V=\frac{|\mathcal{E}|}{2} \delta \varepsilon^{\mathcal{E}}:\left(p^{\mathcal{E}} \boldsymbol{\delta}\right)+\int_{\mathcal{S}_{R}} \frac{1}{2} \delta \boldsymbol{\xi} \cdot \boldsymbol{\sigma} \cdot \boldsymbol{n} d S
$$

Assuming as before that the limit when $R \rightarrow \infty$ of the integral on the external boundary $\mathcal{S}_{R}$ is 0 , it appears that (15) is replaced by

$$
\frac{U(0)-U(\ell)}{\ell}=\frac{|\mathcal{E}|}{2 \ell} \delta \varepsilon^{\mathcal{E}}:\left(\boldsymbol{\sigma}_{0}-p^{\mathcal{E}} \boldsymbol{\delta}\right)
$$

Again, from a strictly mathematical point of view, the existence of a non zero rate of energy release (remember that $|\mathcal{E}| \rightarrow 0$ ) requires the condition $\delta \mathcal{E}^{\mathcal{E}}=O(1 / X)$.

We are now left with the determination of the two unknown quantities: the perturbation strain $\delta \varepsilon^{\mathcal{E}}$ on the one hand, the post-nucleation pore pressure $p^{\mathcal{E}}$ on the other hand, which are obviously strongly coupled to one another.

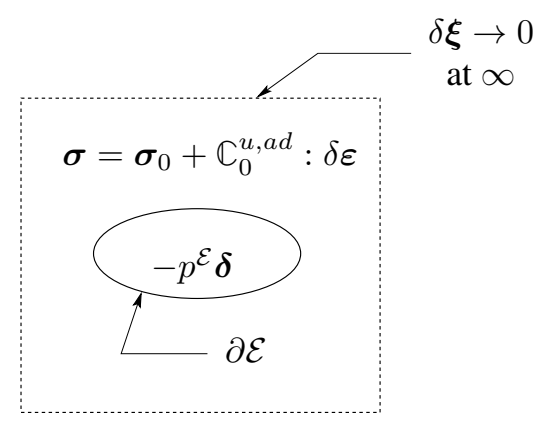

Figure 6 . The post-nucleation mechanical problem in both undrained and adiabatic conditions

A first aspect of this coupling is rather mathematical: the perturbation strain $\delta \mathcal{\varepsilon}^{\mathcal{E}}$ in $\mathcal{E}$ can be derived as the solution to the Eshelby problem depicted at figure 6 as a function of $\sigma_{0}$ and of the post-nucleation pore pressure $p^{\mathcal{E}}$. The boundary conditions at infinity prescribe that the asymptotic perturbation displacement is 0 . As already stated, the stress state inside $\mathcal{E}$ is the spherical stress tensor $-p^{\mathcal{E}} \boldsymbol{\delta}$. It follows that $\delta \mathcal{E}^{\mathcal{E}}$ is obtained from (60) in which $\boldsymbol{E}$ is $0, \boldsymbol{\tau}^{\mathcal{E}}$ is $-p^{\mathcal{E}} \boldsymbol{\delta}, \mathbb{C}^{\mathcal{E}}$ is $0, \mathbb{C}$ is $\mathbb{C}_{0}^{u, a d}$ and $\boldsymbol{\tau}$ is $\boldsymbol{\sigma}_{0}$ :

$$
\delta \mathcal{E}^{\mathcal{E}}=\left(\mathbb{\square}-\mathbb{P}_{0}^{u, a d}: \mathbb{C}_{0}^{u, a d}\right)^{-1}: \mathbb{P}_{0}^{u, a d}:\left(\boldsymbol{\sigma}_{0}+p^{\mathcal{E}} \boldsymbol{\delta}\right)
$$

In the above equation, $\mathbb{P}_{0}^{u, a d}$ is the Hill tensor of $\mathcal{E}$ embedded in the linear elastic medium with stiffness $\mathbb{C}_{0}^{u, a d}$. The second mathematical equation between the two unknowns $\delta \varepsilon^{\mathcal{E}}$ and $p^{\mathcal{E}}$ will now be provided by the state equation of the fluid.

\subsection{The thermoporomechanical coupling}

The nucleation of a crack modelled as a fully damaged zone is expected to induce temperature changes as well as volume and fluid pressure changes. These are the various sides of the thermoporomechanical 
coupling at stake.

Let us begin with the thermomechanical coupling: If we consider the nucleation as an adiabatic phenomenon, the heat produced by the damage process cannot diffuse in the medium at this time scale and is stored in the fully damaged zone $\mathcal{E}$ in the form of internal energy. This implies a temperature increase $\theta$ which is expected to increase the pressure in the fluid filling the pore space of $\mathcal{E}$.

We now consider the poromechanical coupling: nucleation in traction mode a priori induces a crack opening which is associated with a volume increase of the subdomain $\mathcal{E}$. In undrained conditions, $\mathcal{E}$ behaves as a closed system (no fluid mass exchange). We therefore expect that the volume increase will yield a decrease of the fluid pressure.

We observe that the temperature change on the one hand, and the volume change on the other hand have antagonist effects on the fluid pressure. In fact, temperature, volume and fluid pressure are related in the first state equation of the fluid (see (65)). More precisely, let us consider the tangent behavior near the initial state of the fluid (i.e. prior to nucleation). It relates the fluid pressure $p^{\mathcal{E}}$ to the post-nucleation fluid volume $V^{f}$ and the temperature change $\theta$ :

$$
p^{\mathcal{E}}=p_{0}-k_{f}\left(\frac{V^{f}-V_{0}^{f}}{V_{0}^{f}}-3 \alpha_{f} \theta\right)
$$

In the above equation, $V_{0}^{f}$ is the initial volume of the fluid in $\mathcal{E}$, at initial fluid pressure $p_{0}, k_{f}$ is the isothermal fluid compression modulus and $\alpha_{f}$ its thermal expansion coefficient. $V_{f}$ is the current volume of the same fluid mass. Since undrained conditions hold, $V_{f}$ is also the current pore volume. The volume change $V^{f}-V_{0}^{f}$ is therefore proportional to the Lagrangian porosity change (see section 7.3.1):

$$
\frac{V^{f}-V_{0}^{f}}{V_{0}^{f}}=\frac{\phi-\phi_{0}}{\phi_{0}}
$$

It is therefore concluded from a combination of (39) and of the last equation of (62) that:

$$
p^{\mathcal{E}}=p_{0}-M\left(\boldsymbol{B}: \delta \varepsilon^{\mathcal{E}}+L \theta\right)
$$

where $\phi_{0}$ is the initial porosity and $M$ is the Biot modulus (see section 7.3.2):

$$
M=\frac{k_{f} / \phi_{0}}{1+\frac{k_{f} / \phi_{0}}{N}}
$$

In the framework of the assumption of stiff solid phase (see section 7.3.1), it is shown that $\boldsymbol{B}=\boldsymbol{\delta}$ and $L=-3 \bar{\alpha}$. (40) then reduces to

$$
p^{\mathcal{E}}=p_{0}-M\left(\operatorname{tr} \delta \mathcal{\varepsilon}^{\mathcal{E}}-3 \bar{\alpha} \theta\right)
$$

\subsection{A model neglecting the thermal effect}

As a first attempt, it is assumed in the sequel that the effect of the temperature increase on the post-nucleation pressure is negligible. This is, for instance, the case if the medium has a great thermal inertia (i.e. a large heat capacity). In addition, the difference between adiabatic and isothermal stiffness tensors is also neglected and the upper scripts " $\theta$ " or " $a d$ " are therefore omitted:

$$
\mathbb{C}_{0}^{u, a d} \approx \mathbb{C}_{0}^{u, \theta} \approx \mathbb{C}_{0}^{u}=\mathbb{C}_{0}^{d r}+M \boldsymbol{\delta} \otimes \boldsymbol{\delta}
$$

A combination of (38) and (41) (with $\theta \rightarrow 0$ ) yields:

$$
\delta \mathcal{E}^{\mathcal{E}}=\left(\mathbb{\square}-\mathbb{P}_{0}^{u}: \mathbb{C}_{0}^{d r}\right)^{-1}: \mathbb{P}_{0}^{u}:\left(\boldsymbol{\sigma}_{0}+p_{0} \boldsymbol{\delta}\right)
$$

in which it is emphasized that the Hill tensor $\mathbb{P}_{0}^{u}$ refers to the undrained stiffness $\mathbb{C}_{0}^{u}$ and not to $\mathbb{C}_{0}^{d r}$. This makes a very important difference as compared to (10). Indeed, we have previously related the existence of a rate of energy release of order $O\left(X^{0}\right)$ to the property $\delta \varepsilon^{\mathcal{E}}=O(1 / X)$. However, it is found that

$$
\left(\left(\mathbb{\square}-\mathbb{P}_{0}^{u}: \mathbb{C}_{0}^{d r}\right)^{-1}: \mathbb{P}_{0}^{u}\right)_{3333}=\frac{1}{M}+O(X) \quad ; \quad\left(\left(\mathbb{\square}-\mathbb{P}_{0}^{u}: \mathbb{C}_{0}^{d r}\right)^{-1}: \mathbb{P}_{0}^{u}\right)_{33 i i}=O(X) \quad(i=1,2)
$$


We therefore draw an a priori surprising conclusion: at first sight, it seems that a traction in the direction $e_{3}$ (mode I) is not expected to provide a rate of energy release meeting the necessary condition for nucleation. Let us consider for simplicity a loading of the form $\boldsymbol{E}=E \boldsymbol{\delta}$ associated with the spherical stress $\boldsymbol{\sigma}_{0}=\Sigma_{0} \boldsymbol{\delta}$ $\left(\Sigma_{0}=3 k_{0}^{d r} E-p_{0}\right)$. In the nucleation process, the stress inside the subdomain $\mathcal{E}$ starts with the initial value $\boldsymbol{\sigma}_{0}$ and ends up with $-p^{\mathcal{E}} \boldsymbol{\delta}$. However, introducing (42) into (41) (with $\theta \rightarrow 0$ ) yields:

$$
p^{\mathcal{E}}=-\Sigma_{0}+O(X) \quad \Rightarrow \quad \boldsymbol{\sigma}_{0}+p^{\mathcal{E}} \boldsymbol{\delta}=O(X)
$$

In other words, the stress state inside (as well as outside) $\mathcal{E}$ does not vary in the nucleation process, at the leading order in $X$, i.e. $O\left(X^{0}\right)$. This is the reason why it induces neither large strain nor energy release. More precisely, it is found that;

$$
\frac{U(0)-U(\ell)}{\ell}=\frac{4 a X}{3 M} \Sigma_{0}\left(\Sigma_{0}+p_{0}\right)+O\left(X^{2}\right)
$$

which should be compared to (19). The order $O(X)$ in the expression of $\boldsymbol{\sigma}_{0}+p^{\mathcal{E}} \boldsymbol{\delta}$ is governing the perturbation strain $\delta \varepsilon^{\mathcal{E}}$ (see (38)):

$$
\delta \mathcal{\varepsilon}^{\mathcal{E}}=3 E \frac{k_{0}^{d r}}{M} \boldsymbol{e}_{3} \otimes \boldsymbol{e}_{3}+O(X)
$$

In this form, the perturbation strain is a priori of the order of $E$. In fact, the physical mechanism that inhibits the nucleation is based on a stress transfer from the porous material to the fluid alone and relies on the stiffness of the latter which is characterized by $M$. In contrast, if the fluid is very compressible, then $M \ll k_{0}^{d r}$ and the perturbation strain is not infinitesimal: in this case, the previous conclusion is no longer valid and nucleation in mode I is expected to take place.

While this paper is devoted to mode I nucleation, let us briefly consider a shear loading

$$
\boldsymbol{E}=E\left(\boldsymbol{e}_{1} \otimes \boldsymbol{e}_{3}+\boldsymbol{e}_{3} \otimes \boldsymbol{e}_{1}\right)
$$

We therefore have $\boldsymbol{\sigma}_{0}+p_{0} \boldsymbol{\delta}=2 \mu_{0} \boldsymbol{E}$. It is found that

$$
\left(\left(\mathbb{\square}-\mathbb{P}_{0}^{u}: \mathbb{C}_{0}^{d r}\right)^{-1}: \mathbb{P}_{0}^{u}\right)_{1313}=\frac{\gamma}{X}+O\left(X^{0}\right) \quad \text { with } \quad \gamma=\frac{3 k_{0}^{u}+4 \mu_{0}}{3 \pi \mu_{0}\left(3 k_{0}^{u}+2 \mu_{0}\right)}
$$

and accordingly

$$
\delta \varepsilon^{\mathcal{E}}=\frac{4 \mu_{0} \gamma E}{X}\left(\boldsymbol{e}_{1} \otimes \boldsymbol{e}_{3}+\boldsymbol{e}_{3} \otimes \boldsymbol{e}_{1}\right)
$$

This kind of loading does not induce any pressure change in the subdomain $\mathcal{E}$ which reacts as if it were empty. The condition $\delta \varepsilon^{\mathcal{E}}=O(1 / X)$ for nucleation is fullfilled. This emphasizes the fact that the previously stated conclusion was in fact strictly devoted to mode I. The latter will now be discussed into detail.

\section{DISCUSSION}

In this section, we discuss the conclusion drawn in the case of the isotropic loading $\boldsymbol{E}=E \boldsymbol{\delta}$. We keep in mind that mode I is associated with $E>0$ (see (46)) and $\Sigma_{0}+p_{0}=3 k_{0}^{d r} E>0$. The starting point is the estimate (44) of the fluid pressure in the crack. Since $E>0$, we first observe that the final pressure $p^{\mathcal{E}}$ is always smaller than the initial one $p_{0}$, which is consistent with the idea of a fluid pressure decrease during nucleation (figure 7).

First, assume that $\Sigma_{0}<0$ (whereas $\Sigma_{0}+p_{0}>0$ ). (45) then indicates that the energy release is not only of the order of $X$ (that is, vanishing when $X \rightarrow 0$ ), but also is a negative quantity. Clearly enough, this means that a brittle failure mechanism of nucleation in mode I (for some strain level $E$ ) is impossible in the case of a negative confinement $\left(\sigma_{0}=\Sigma_{0} \delta\right)$. This conclusion questions the use of an effective stress-based nucleation criterion.

We now focus on the case $\Sigma_{0}>0$. The estimate (44) of the fluid pressure in the crack would then be negative (figure 7). However, such a scenario is not physically relevant. Indeed, in the case of full saturation, 


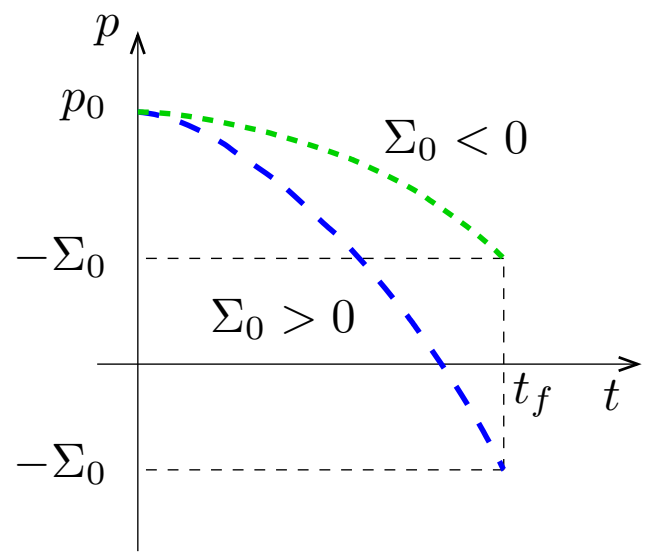

Figure 7. Evolution of the fluid pressure in the crack: possible scenarios

the sign of the liquid pressure is necessarily positive since it is lower bounded by the saturation vapour pressure. Note that this would not be the case in the situation of partial saturation. Indeed, according to Kelvin's law, in the equilibrium of a liquid with its vapour, a negative liquid pressure can be encountered at a given vapour pressure due to capillary tension effects and provided that the relative humidity be low enough. If we follow the pressure decrease in the phase diagram of the fluid (figure 8), a (partial) vaporization of the liquid is expected to take place when the liquid pressure reaches the saturation vapour pressure. This change of phase implies that the fluid looses its stiffness which was accounted for by the Biot modulus $M$. This amounts to say that we are brought back to the non porous case studied at section 3.3. The conclusion of section 3.3.2 (unstable damage) indicates that the partial vaporization triggers a brittle failure mechanism in which the crack is nucleated. Still, nucleation in the saturated material cannot be termed a brittle mechanism if the strain level $E$ at which the vaporization takes place is higher than the elastic threshold at which damage is initiated. The presence of the fluid in the pore space is therefore responsible for a ductile phase in the nucleation mechanism. Two questions arise at that point.

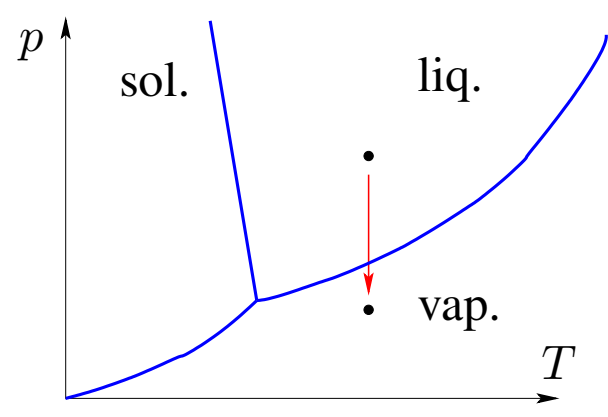

Figure 8. Phase diagram of water

- Is it possible to model the damage process from the elastic threshold until the vaporization?

- What is the final thermodynamic state of the fluid at the end of the nucleation ?

\subsection{Modeling the damage process}

Let $\boldsymbol{E}^{e l}$ denote the strain level at the onset of damage. During the damage process, the stress in the damaged zone, the strain and the fluid pressure are related by a state equation:

$$
\boldsymbol{\sigma}^{\mathcal{E}}=\mathbb{C}^{d r}(d): \varepsilon^{\mathcal{E}}-p^{\mathcal{E}} \boldsymbol{\delta}
$$

With respect to the first equation of (62) (with $\theta \rightarrow 0$ ), the Biot tensor $\boldsymbol{B}$ in the above equation is taken equal to $\boldsymbol{\delta}$ (see (64)). The damage thus affects the effective drained tensor $\mathbb{C}^{d r}(d)$ only. Rigorously speaking, note 
that this conclusion requires that the Biot tensor of the sound material be itself equal to $\delta$ which amounts to the assumption of stiff solid phase $\left|\mathbb{C}_{s}^{-1}: \mathbb{C}^{d r}(0)\right| \ll 1$ (see section 7). During an undrained evolution, the pressure change is controlled by the undrained constraint (66). The above state equation therefore yields:

$$
\boldsymbol{\sigma}^{\mathcal{E}}=\mathbb{C}^{u}(d): \varepsilon^{\mathcal{E}}+\boldsymbol{\tau}_{0} \quad \text { with } \quad \boldsymbol{\tau}_{0}=-p_{0} \boldsymbol{\delta}-M \boldsymbol{\delta} \otimes \boldsymbol{\delta}: \boldsymbol{E}^{e l}
$$

in which the undrained stiffness tensor reads:

$$
\mathbb{C}^{u}(d)=\mathbb{C}^{d r}(d)+M \boldsymbol{\delta} \otimes \boldsymbol{\delta}
$$

We herein consider a class of isotropic damage models in which $\mathbb{C}^{d r}(d)=3 k^{d r}(d) \mathbb{\downarrow}+2 \mu(d) \mathbb{K}$ with:

$$
\frac{k^{d r}(d)}{k_{0}^{d r}}=\frac{\mu(d)}{\mu_{0}}=f(d) \quad \text { with } \quad f(d)=\frac{1-Q d}{1+Q^{\prime} d}
$$

with $Q, Q^{\prime}>0$. This model is formally identical to (35), except that $\mathbb{C}$ is replaced by the drained stiffness tensor $\mathbb{C}^{d r}$. For practical applications and numerical simulations, $f(d)$ will be chosen as the ratio $k^{d r}(d) / k_{0}^{d r}$ predicted by the Ponte-Castaneda-Willis homogenization scheme [13] for an isotropic solid damaged by a network of microcracks with isotropic distribution of orientations:

$$
Q=\frac{32}{27}\left(1+\nu_{0}^{d r}\right) \quad ; \quad Q^{\prime}=\frac{16}{27} \frac{\left(1+\nu_{0}^{d r}\right)^{2}}{1-2 \nu_{0}^{d r}}
$$

The very important feature in (48) is that the undrained bulk modulus $k^{u}$ now comprises a component that is not subjected to damage:

$$
k^{u}(d)=k^{d r}(d)+M
$$

Out of the domain $\mathcal{E}$, the state equation involves the sound undrained stiffness tensor $\mathbb{C}_{0}^{u}=\mathbb{C}^{u}(0)$ :

$$
z \in \Omega \backslash \mathcal{E}: \quad \sigma=\mathbb{C}_{0}^{u}: \varepsilon+\tau_{0}
$$

Since $\tau_{0}$ is a constant, (47) shows that the damage process in an undrained material is formally identical to that of a non porous material. The two fundamental equations (25) and (26) which control the damage process were already presented at section 3.3 where the non porous (or dry) case was considered. They are still valid in an undrained evolution of a saturated porous material provided that $\mathbb{C}(d)$ is replaced by $\mathbb{C}^{u}(d)$. At the strain level when the damage process is initiated, the strain field is still uniform so that the damage criterion (25) reads

$$
-\frac{1}{2} \boldsymbol{E}^{e l}: \mathbb{C}^{u \prime}(d): \boldsymbol{E}^{e l}=g_{c}
$$

which yields the elastic threshold $E=E^{e l}$ in the form:

$$
E^{e l}=\frac{1}{3} \sqrt{-\frac{2 g_{c}}{\partial k^{d r} / \partial d(0)}} \quad \text { with } \quad \frac{\partial k^{d r}}{\partial d}(0)=-\left(Q+Q^{\prime}\right) k_{0}^{d r}
$$

Beyond the threshold $E^{e l}$, we need to determine the 3 unknowns, namely the strain tensor $\varepsilon^{\mathcal{E}}$, the damage level $d$ and the fluid pressure $p^{\mathcal{E}}$. The first equation is provided by the damage criterion:

$$
-\frac{1}{2} \varepsilon^{\mathcal{E}}: \mathbb{C}^{u^{\prime}}(d): \varepsilon^{\mathcal{E}}=g_{c}
$$

We then observe from (47) and (50) that $\varepsilon^{\mathcal{E}}$ is the solution of an Eshelby problem (see (60)):

$$
\varepsilon^{\mathcal{E}}=\left(\mathbb{0}+\mathbb{P}_{0}^{u}:\left(\mathbb{C}^{u}(d)-\mathbb{C}_{0}^{u}\right)\right)^{-1}: \boldsymbol{E}
$$

Eliminating $\varepsilon^{\mathcal{E}}$, these two equations can be solved with respect to $\boldsymbol{E}$ and $d$. Finally, the fluid pressure is derived from the undrained constraint (66):

$$
p=p_{0}-M \boldsymbol{\delta}:\left(\varepsilon^{\mathcal{E}}-\boldsymbol{E}^{e l}\right)
$$




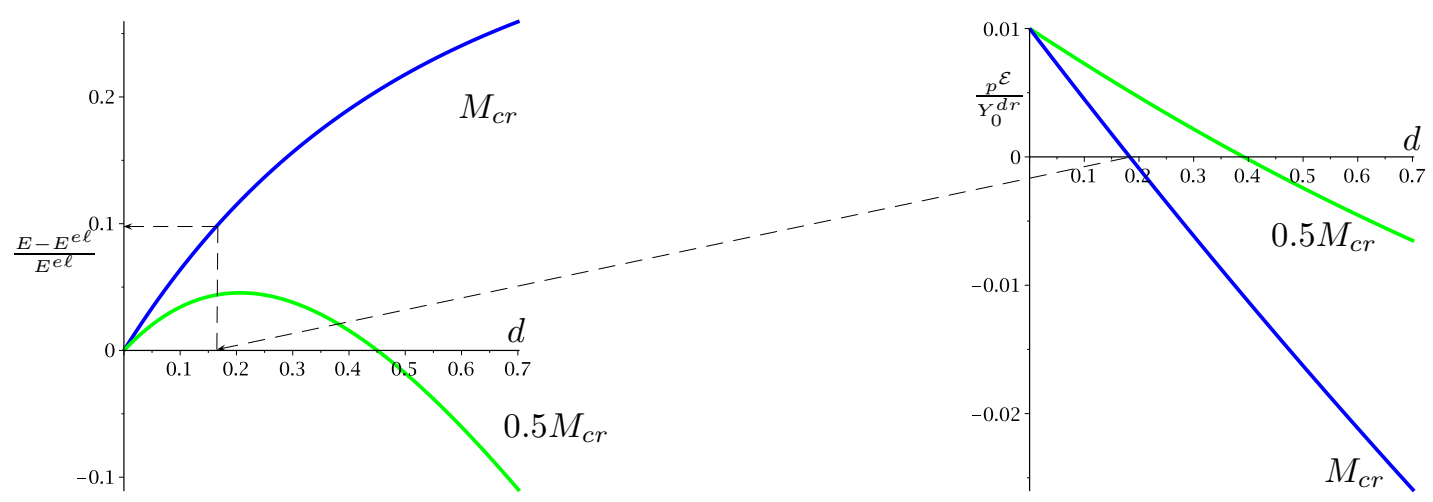

Figure 9. Asymptotic strain $E$ and crack fluid pressure $p^{\mathcal{E}}$ as functions of the damage parameter $d . g_{c} / Y_{0}^{d r}=10^{-3}$, $\nu_{0}^{d r}=0.2, p_{0} / Y_{0}^{d r}=10^{-2}$

The numerical simulations require numerical values for the ratios $g_{c} / Y_{0}^{d r}$ and $M / Y_{0}^{d r}\left(Y_{0}^{d r}\right.$ is the drained Young modulus of the sound material), and for its Poisson coefficient $\nu_{0}^{d r}$. For further use, we introduce the critical Biot modulus $M_{c r}$ defined as:

$$
M_{c r}=\left(k_{0}^{d r}+\frac{4}{3} \mu_{0}^{d r}\right) \frac{Q}{Q^{\prime}}=2 Y_{0}^{d r} \frac{\left(1-\nu_{0}^{d r}\right)}{\left(1+\nu_{0}^{d r}\right)^{2}}
$$

Typical results are presented at figure 9 for two values of $M$, respectively $M_{c r}$ and $0.5 M_{c r}$. For $M=$ $0.5 M_{c r}$, the asymptotic strain $E$ is not a monotonic function of the damage parameter. This indicates that the response of the system is not stable. In contrast, for $M=M_{c r}$, the asymptotic strain $E$ is a strictly increasing function of the damage parameter. This suggests that the response could be stable. If this is confirmed, these plots can be used to determine the damage level at which the fluid pressure reaches the saturation vapour pressure and the corresponding strain level, as indicated at figure 9 . In order to clarify the role of the Biot modulus on the stability of the damage process, we now apply Hill's criterion which was already presented at section 3.3. In the expressions (27)-(28) of the tangent stiffness tensor $\mathbb{C}_{t}^{u}$ in undrained conditions, $\mathbb{C}(d)$ is replaced by $\mathbb{C}^{u}(d)$. In the non damaged zone, the tangent tensor $\mathbb{C}_{t}^{u}$ is therefore equal to $\mathbb{C}_{0}^{u}$.

If shear modes are disregarded, the discussion concerning the positiveness of the tensor $\mathbb{P}_{0}^{u-1}-\mathbb{C}_{0}^{u}+\mathbb{C}_{t}^{u}$ amounts to the sign of the eigenvalue $\lambda_{I}$ corresponding to mode I. At the leading order $\left(X^{0}\right)$, it is found that (see section 7):

$$
\lambda_{I}(\varepsilon, d)=M-2 \frac{f^{\prime 2}(d)}{f^{\prime \prime}(d)} \frac{\left(\varepsilon: \mathbb{C}^{d r}(0): \boldsymbol{E}_{3}\right)^{2}}{\varepsilon: \mathbb{C}^{d r}(0): \varepsilon}+f(d) C^{d r}(0)_{3333}+O(X) \quad \text { with } \quad \boldsymbol{E}_{3}=\boldsymbol{e}_{3} \otimes \boldsymbol{e}_{3}
$$

At a given damage level, the minimum of $\lambda_{I}(\varepsilon, d)$ with respect to $\varepsilon$ is reached at $\varepsilon=\boldsymbol{E}_{3}$ and proves to be independant on $d$ (see again section 7):

$$
\lambda_{\text {Imin }}=M-C^{d r}(0)_{3333} \frac{Q}{Q^{\prime}}
$$

The unconditional positiveness of $\lambda_{I}$ (which is the condition of stability provided by Hill's criterion) thus amounts to $M>M_{c r}$ (see (52)).

\subsection{Thermodynamical analysis of the post nucleation fluid state}

As stated before, nucleation in the case $M>M_{c r}$ requires a partial vaporization of the fluid that fills the domain $\mathcal{E}$ (total fluid mass $m$ ). The thermodynamical state of the fluid after nucleation is characterized by the mass fraction $x_{v}$ of the vapour (resp. $x_{\ell}=1-x_{v}$ of the liquid) in the fluid mixture and its temperature $T^{*}$ which is a priori different from the temperature $T_{0}$ at the initiation of the vaporization (see figure 10). 


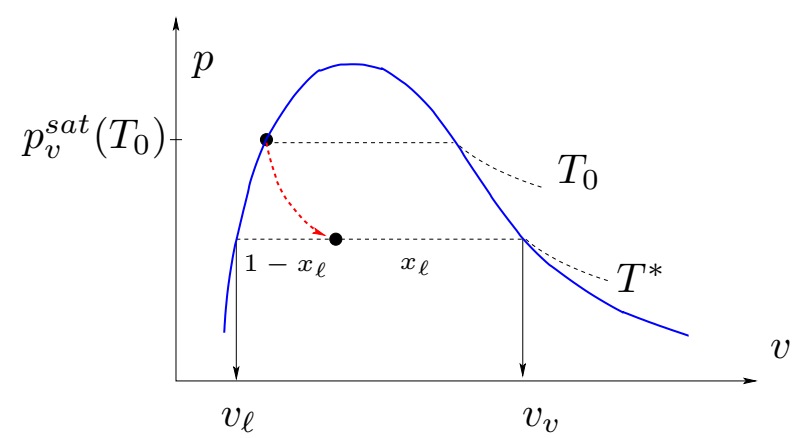

Figure 10. Change of physical state: representation in the $(v, p)$ plane

Let $s_{\ell}$ (resp. $s_{v}$ ) denote the entropy of the mass unit of liquid (resp. vapour). The conservation of entropy during the vaporization under adiabatic condition provides a first equation between the two unknowns $x_{\ell}$ and $T^{*}$ :

$$
m\left(s_{\ell}\left(T^{*}\right) x_{\ell}+s_{v}\left(T^{*}\right)\left(1-x_{\ell}\right)\right)=m s_{\ell}\left(T_{0}\right)
$$

where $m$ is the fluid mass in the initial volume $V_{c r}^{0}$ of the crack, that is $m=\rho_{\ell} \phi_{0} V_{c r}^{0}$. A second one accounts for the undrained constraint. It expresses that the post-nucleation volume $V_{c r}$ of the nucleated crack is filled with the (fully damaged) solid, the liquid and the vapour. In practice, the specific volume $v_{v}$ of the vapour is at least 3 orders of magnitude larger:

$$
V_{c r}=V_{s o l}+m\left(v_{\ell}\left(T^{*}\right) x_{\ell}+v_{v}\left(T^{*}\right)\left(1-x_{\ell}\right)\right) \approx m v_{v}\left(T^{*}\right)\left(1-x_{\ell}\right)
$$

In the above equation, $V_{c r}$ is still an unknown. The crack volume change $\delta V_{c r}$ is the sum of the respective contributions of the stable damage process $\left(p>p_{v}^{s a t}\left(T_{0}\right)\right)$ and of the brittle failure following the partial vaporization of the fluid. During the whole process, the asymptotic strain $E$ increases from $E^{e l}$ to $E^{*}$ while the stress state in the crack simultaneously drops from $\boldsymbol{\sigma}_{0}$ to $-p_{v}^{\text {sat }}\left(T^{*}\right) \boldsymbol{\delta}$ (see figure 11). In view of determining an order of magnitude of the vapour mass fraction $x_{v}$ and of the temperature change, it seems reasonable to make the approximation $E^{e l} \approx E^{*}$ and to focus on the effect of the stress state change in the crack. A new application of (60) then yields:

$$
\frac{\delta V_{c r}}{V_{c r}^{0}}=\frac{1}{X_{0}} \boldsymbol{\delta}: \mathbb{Q}_{0}^{u}:\left(\boldsymbol{\sigma}_{0}+p_{v}\left(T^{*}\right) \boldsymbol{\delta}\right) \quad \text { with } \quad \mathbb{Q}_{0}^{u}=\lim _{X \rightarrow 0} X\left(\mathbb{\square}-\mathbb{P}_{0}^{u}: \mathbb{C}_{0}^{u}\right): \mathbb{P}_{0}^{u}
$$

where $X_{0}$ is the initial aspect ratio, that is, prior to nucleation. The three above equations provide the framework for the determination of the physical post-nucleation state (see also section 7.4). In practice, (53)-(54) can be solved with respect to $x_{\ell}$ and $T^{*}$ provided that $\delta V_{c r} / V_{c r}^{0}$ is given. For $X_{0}$ of the order of $10^{-5}$, and using typical material constants of argilite, $x_{v}$ and $\left(T^{*}-T_{0}\right) / T_{0}$ are found to be of the order of several \% while $\delta V_{c r} / V_{c r}^{0}$ is of several hundreds. Despite the fact that the vaporized mass fraction is infinitesimal, the large strain induced by nucleation is accomodated by the vapour due to its very large specific volume.

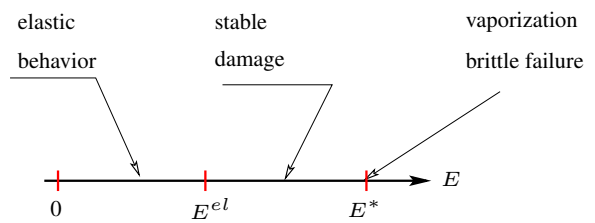

Figure 11. Delayed brittle response in the case $M>M_{c r}$ 


\section{CONCLUDING REMARKS}

The central role of the Biot modulus in the discussion on the stability of the damage process deserves that its micromechanical interpretation be recalled (see section 7.3.2). In the assumption of stiff solid phase:

$$
\frac{1}{M}=\frac{1-\phi_{0}}{k_{s}}+\frac{\phi_{0}}{k_{f}}
$$

where $k_{s}$ is the compression modulus of the solid at the microcopic scale and $k_{f}$ that of the fluid. In water saturated rocks, the property $k_{s} \gg k_{f}$ allows to approximate $M$ by the ratio $k_{f} / \phi_{0}$, so that the numerical value of $M$ is related to the fluid compressibility. As already mentioned, in the case of a partial vaporization, the liquid is replaced by the much more compressible fluid mixture and the Biot modulus dramatically drops.

\begin{tabular}{ccc} 
high compr. & low compr. \\
\hline $\begin{array}{c}\text { negligible } \\
\text { fluid effect }\end{array}$ & $M_{c r}$ & $\begin{array}{c}\text { delayed } \\
\text { brittleness }\end{array}$
\end{tabular}

Figure 12 . The role of $M$ on the stability of the damage process

The conclusions of the discussion presented in section 5 can be summarized by the scheme of figure 12. If $M<M_{c r}$ (high fluid compressibility), a non stable damage process (modelled according to the principles of the damage theory of section 3.3.1) is obtained. This strongly suggests that a brittle failure mechanism akin to the non porous case takes place at or shortly after the initiation of damage $\left(E=E^{e l}\right)$. In this case, the fluid role is negligible. In contrast, if $M>M_{c r}$, the damage process is stable as long as the condition for vaporization is not reached, that is as long as the liquid pressure $p$ is greater than the saturation vapour pressure $p_{v}^{\text {sat }}$. The condition for physical phase change $p=p_{v}^{\text {sat }}$ corresponds to a second threshold $E^{*}>E^{e l}$. At this instant, a brittle failure mechanism is possible due to the dramatic increase of fluid compressibility. Nevertheless, the existence of a stable loading interval $E \in\left[E^{e l}, E^{*}\right]$ represents a delay in the material brittleness (see figure 11).

\section{APPENDIX}

\subsection{Eigenvalues of $\mathbb{P}_{0}^{u-1}-\mathbb{C}_{0}^{u}+\mathbb{C}_{t}^{u}$}

We herein consider a class of isotropic damage models in which $\mathbb{C}^{u}(d)=3\left(k^{d r}(d)+M\right) \mathbb{}+2 \mu(d) \mathbb{K}$ with:

$$
\frac{k(d)}{k_{0}}=\frac{\mu(d)}{\mu_{0}}=f(d) \quad \text { with } \quad f(d)=\frac{1-Q d}{1+Q^{\prime} d}
$$

In the non porous case, we have $M=0$ (and then $\mathbb{P}_{0}^{u} \equiv \mathbb{P}_{0}, \mathbb{C}_{0}^{u} \equiv \mathbb{C}_{0}$ ). In the undrained saturated case, $M$ is the (constant) Biot modulus while $k_{0}=k_{0}^{d r}$. In the latter case, $M$ does not depend on $d$.

We now consider a flat spheroid with symmetry axis along $\boldsymbol{e}_{3}$ (aspect ratio $X$ ) and the Hill's tensor $\mathbb{P}_{0}^{u}$ associated with the stiffness tensor $\mathbb{C}(0)+M \boldsymbol{\delta} \otimes \boldsymbol{\delta}$ of the isotropic sound material (bulk and shear moduli $k_{0}^{u}=k_{0}+M$ and $\left.\mu_{0}\right) . \mathbb{P}_{0}^{u-1}-\mathbb{C}_{0}^{u}$ is a transverse isotropic tensor w.r.t. the symmetry axis along $\boldsymbol{e}_{3}$. The $6 \times 6$ Voigt matrix $V$ of such a transverse isotropic 4 -order tensor $\vee$ reads:

$$
V=\left(\begin{array}{ll}
A & 0 \\
0 & B
\end{array}\right)
$$

where $A$ and $B$ are $3 \times 3$ matrices. By definition (other coefficients are equal to 0 ):

$$
A_{i j}=V_{i i j j} \quad ; \quad B_{11}=2 V_{2323} \quad ; \quad B_{22}=2 V_{1313} \quad ; \quad B_{33}=2 V_{1212}
$$


The eigenvalues of $V$ are the $B_{i i}(i=1,2,3)$ as well as the eigenvalues of $A$. At the leading order w.r.t. $X$, the matrix $A$ corresponding to $\mathbb{P}_{0}^{u-1}-\mathbb{C}_{0}^{u}$ reads:

$$
A_{0}=\left(\begin{array}{ccc}
\frac{\mu_{0} a_{0}}{X} & \frac{\mu_{0} b_{0}}{X} & c_{0} \\
\frac{\mu_{0} b_{0}}{X} & \frac{\mu_{0} a_{0}}{X} & c_{0} \\
c_{0} & c_{0} & e_{0} X
\end{array}\right)
$$

where $\mu_{0}$ is the shear modulus while $a_{0}, b_{0}, c_{0}$ and $e_{0}$ are the following functions of $k_{0}, M$ and $\mu_{0}$ $\left(k_{0}^{u}=k_{0}+M\right)$ :

$a_{0}=\frac{4\left(15 k_{0}^{u}+29 \mu_{0}\right)\left(3 k_{0}^{u}+4 \mu_{0}\right)}{3 \pi\left(3 k_{0}^{u}+7 \mu_{0}\right)\left(3 k_{0}^{u}+5 \mu_{0}\right)} ; b_{0}=\frac{4\left(3 k_{0}^{u}+\mu_{0}\right)\left(3 k_{0}^{u}+4 \mu_{0}\right)}{3 \pi\left(3 k_{0}^{u}+7 \mu_{0}\right)\left(3 k_{0}^{u}+5 \mu_{0}\right)} ; c_{0}=\frac{6 \mu_{0}^{2}}{3 k_{0}^{u}+7 \mu_{0}} ; e_{0}=\frac{\pi \mu_{0}\left(3 k_{0}^{u}+4 \mu_{0}\right)}{3 k_{0}^{u}+7 \mu_{0}}$

We now consider the tangent stiffness tensor. Recalling (56), (27) yields:

$$
\mathbb{C}_{t}^{u}=f(d) \mathbb{C}(0)+M \boldsymbol{\delta} \otimes \boldsymbol{\delta}-2 \frac{f^{\prime 2}(d)}{f^{\prime \prime}(d)} \frac{(\mathbb{C}(0): \varepsilon) \otimes(\mathbb{C}(0): \varepsilon)}{\varepsilon: \mathbb{C}(0): \varepsilon}
$$

Since we focus on instability in mode I (crack opening in the direction of $e_{3}$ ), it is assumed in the following that $\varepsilon^{\mathcal{E}}$ belongs to the vector space generated by $\boldsymbol{e}_{i} \otimes \boldsymbol{e}_{i}(i=1,2,3) . \mathbb{C}(0)$ being an isotropic tensor, this implies that the Voigt matrix of $\mathbb{C}_{t}$ has the structure of (57). The same property also holds for the Voigt matrix of $\mathbb{P}_{0}^{u-1}-\mathbb{C}_{0}^{u}+\mathbb{C}_{t}^{u}$. At the leading order w.r.t. $X$, the block $A$ of the latter reads:

$$
A=\left(\begin{array}{ccc}
\frac{\mu_{0} a_{0}}{X} & \frac{\mu_{0} b_{0}}{X} & c \\
\frac{\mu_{0} b_{0}}{X} & \frac{\mu_{0} a_{0}}{X} & c \\
c & c & e
\end{array}\right)
$$

where $e=C_{t 3333}^{u}$. Only the third line (resp. column) has been modified at the leading order. The discussion on the instability in mode I amounts to the determination of the eigenvalues of the above matrix $A$ and their signs. They are the roots $\lambda$ of the characteristic polynom:

$$
\chi_{A}(\lambda)=\left(C_{t 3333}^{u}-\lambda\right)\left(\left(\mu_{0} \frac{a_{0}-b_{0}}{X}-\lambda\right)^{2}+2 \frac{\mu_{0} b_{0}}{\lambda}\left(\mu_{0} \frac{a_{0}-b_{0}}{X}-\lambda\right)\right)-2 c^{2}\left(\mu_{0} \frac{a_{0}-b_{0}}{X}-\lambda\right)
$$

The asymptotic analysis yields 3 distinct roots. At the leading order w.r.t. $X$, they read:

$$
\lambda_{1}=C_{t 3333}^{u} \quad ; \quad \lambda_{2}=\mu_{0} \frac{a_{0}-b_{0}}{X} \quad ; \quad \lambda_{3}=\mu_{0} \frac{a_{0}+b_{0}}{X}
$$

Since $a_{0}>b_{0}>0$, it appears that $0<\lambda_{2}<\lambda_{3}$. The stability condition provided by Hill's criterion thus reads $C_{t 3333}^{u}>0$ which takes the form of a condition on the Biot modulus:

$$
M>2 \frac{f^{\prime 2}(d)}{f^{\prime \prime}(d)} \frac{\left(\varepsilon: \mathbb{C}(0): \boldsymbol{E}_{3}\right)^{2}}{\varepsilon: \mathbb{C}(0): \boldsymbol{\varepsilon}}-f(d) C(0)_{3333}
$$

where $\boldsymbol{E}_{3}=\boldsymbol{e}_{3} \otimes \boldsymbol{e}_{3}$. It appears that this condition seemingly depends on the strain state $\varepsilon$ and on the damage state $d$. Still, let us introduce the scalar product on the vector space $\mathcal{S}_{2}$ of symmetric second order tensors, defined as:

$$
(\boldsymbol{a} \mid \boldsymbol{b})=\boldsymbol{a}: \mathbb{C}(0): \boldsymbol{b}
$$

A direct application of Cauchy-Schwarz inequality yields:

$$
\max _{\boldsymbol{\varepsilon} \in \mathcal{S}_{2}} \frac{\left(\varepsilon: \mathbb{C}(0): \boldsymbol{E}_{3}\right)^{2}}{\varepsilon: \mathbb{C}(0): \varepsilon}=\boldsymbol{E}_{3}: \mathbb{C}(0): \boldsymbol{E}_{3}=C(0)_{3333}
$$


Besides, the rational function $f(d)$ has the following property:

$$
2 \frac{f^{\prime 2}(d)}{f^{\prime \prime}(d)}-f(d)=\frac{Q}{Q^{\prime}}
$$

Eventually, Hill's criterion provides a very simple (sufficient) stability condition on the Biot modulus, which is independant of the current state $(d, \varepsilon)$ :

$$
M>C(0)_{3333} \frac{Q}{Q^{\prime}}
$$

\subsection{The generalized Eshelby problem}

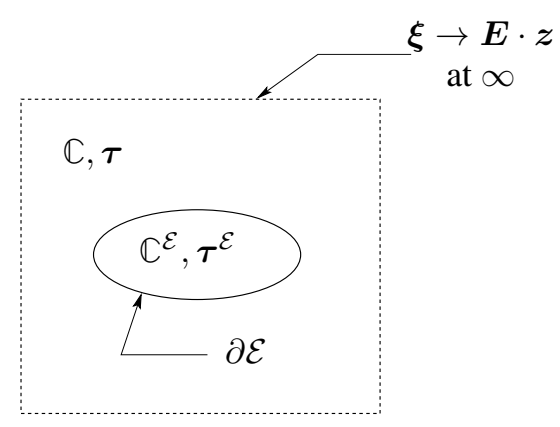

Figure 13. The generalized Eshelby problem

Consider an infinite homogeneous linear elastic medium with stiffness tensor $\mathbb{C}$ in which the constitutive equation reads:

$$
\sigma=\mathbb{C}: \epsilon+\tau
$$

The tensor $\tau$ represents some uniform initial stress in the reference state. An ellipsoidal (resp. elliptic in plane strain conditions) domain $\mathcal{E}$ is embedded in this medium. The constitutive equation in $\mathcal{E}$ reads (uniform initial stress $\tau^{\mathcal{E}}$ ):

$$
\sigma=\mathbb{C}^{\mathcal{E}}: \epsilon+\tau^{\mathcal{E}}
$$

Under application of an asymptotic uniform strain $\boldsymbol{E}$ at infinity (figure 13), the strain induced in the domain $\mathcal{E}$ is uniform and reads (e.g. [3]):

$$
\boldsymbol{\epsilon}^{\mathcal{E}}=\left(\mathbb{\square}+\mathbb{P}:\left(\mathbb{C}^{\mathcal{E}}-\mathbb{C}\right)\right)^{-1}:\left(\boldsymbol{E}-\mathbb{P}:\left(\boldsymbol{\tau}^{\mathcal{E}}-\boldsymbol{\tau}\right)\right)
$$

where $\mathbb{P}$ is the Hill's tensor of $\mathcal{E}$ embedded in the homogenous medium with stiffness tensor $\mathbb{C}$.

\subsection{Fundamentals of thermoporoelasticity}

The state equations of thermoporoelasticity are derived either from an appropriate macroscopic thermodynamic framework (e.g. [16]) or from a micromechanics reasoning ((e.g. [4]). The second way is briefly recalled hereafter. In this section, the physical quantities at the microscopic (resp. macroscopic) scale are denoted by lower case (resp. upper case) letters. The subscript 0 refers to the initial configuration.

7.3.1. State equations Consider a representative elementary volume $\Omega$ (r.e.v.) of a porous material, made up of a solid phase $\Omega_{s}$ and a fluid phase $\Omega_{f}$. For any field $a(\boldsymbol{z})$ at the microscopic field, the following averages will be used:

$$
\bar{a}=\frac{1}{\left|\Omega_{0}\right|} \int_{\Omega} a(\boldsymbol{z}) d V \quad ; \quad \bar{a}^{\alpha}=\frac{1}{\left|\Omega_{\alpha, 0}\right|} \int_{\Omega_{\alpha}} a(\boldsymbol{z}) d V
$$

At the microscopic scale, the solid material (subscript $s$ ) is linear thermoelastic:

$$
\begin{aligned}
& \boldsymbol{\sigma}=\boldsymbol{\sigma}_{0}+\mathbb{C}_{s}: \varepsilon-\boldsymbol{\kappa}_{s} \theta \\
& s_{s}=s_{s}^{0}+c_{s} \theta+\boldsymbol{\kappa}_{s}: \varepsilon
\end{aligned}
$$


where $\sigma$ and $\varepsilon$ are the stress and strain fields while $s_{s}$ is the volume density of entropy in the solid phase $\Omega_{s}$. The temperature change $\theta$ is uniform at the scale of the r.e.v. and the fluid pressure $p$ is also uniform throughout the pore space $\Omega_{f}$. Besides, Hashin boundary conditions on the microscopic displacement field $\boldsymbol{\xi}$ hold at any point $\boldsymbol{z}$ on the boundary $\partial \Omega$ of the r.e.v.: $\boldsymbol{\xi}(\boldsymbol{z})=\boldsymbol{E} \cdot \boldsymbol{z}$ where $\boldsymbol{E}$ is the macroscopic strain tensor applied to the r.e.v., related to the strain field by the average rule $\boldsymbol{E}=\overline{\boldsymbol{\varepsilon}}$.

Let $S_{s}=\left(1-\phi_{0}\right){\overline{s_{s}}}^{s}$ and $\boldsymbol{\Sigma}=\overline{\boldsymbol{\sigma}}$ respectively denote the macroscopic density of entropy of the solid the macroscopic stress tensor. Let $\phi$ denote the Lagrangian porosity, defined as the pore volume normalized by $\left|\Omega_{0}\right|$; the normalized pore volume change is $\phi-\phi_{0}=\phi_{0} \overline{\operatorname{tr}} \varepsilon^{f}$. The macroscopic state equations are derived from the microscopic state equations (61) with the average rules:

$$
\begin{aligned}
& \boldsymbol{\Sigma}=\boldsymbol{\Sigma}_{0}+\mathbb{C}^{d r, \theta}: \boldsymbol{E}-\boldsymbol{B}\left(p-p_{0}\right)-\boldsymbol{K} \theta \\
& S_{s}=S_{s}^{0}+\boldsymbol{K}: \boldsymbol{E}+L_{s}\left(p-p_{0}\right)+C_{s} \theta \\
& \phi-\phi_{0}=\boldsymbol{B}: \boldsymbol{E}+\frac{p-p_{0}}{N}+L_{s} \theta
\end{aligned}
$$

$\mathbb{C}^{d r, \theta}$ is the homogenized stiffness tensor in both drained and isothermal conditions. The scalar and tensorial coefficients appearing in these equations are related to $\mathbb{C}^{d r, \theta}$ and to the microscopic coefficients by the following relationships:

$$
\begin{aligned}
& \boldsymbol{B}=\boldsymbol{\delta}:\left(\mathbb{\square}-\mathbb{C}_{s}^{-1}: \mathbb{C}^{d r, \theta}\right) \\
& \boldsymbol{K}=\boldsymbol{\kappa}_{s}: \mathbb{C}_{s}^{-1}: \mathbb{C}^{d r, \theta} \\
& L_{s}=-\boldsymbol{\kappa}_{s}: \mathbb{C}_{s}^{-1}:\left(\boldsymbol{B}-\phi_{0} \boldsymbol{\delta}\right) \\
& 1 / N=\boldsymbol{\delta}: \mathbb{C}_{s}^{-1}:\left(\boldsymbol{B}-\phi_{0} \boldsymbol{\delta}\right) \\
& C_{s}=\left(1-\phi_{0}\right) c_{s}+\boldsymbol{\kappa}_{s}: \mathbb{C}_{s}^{-1}:\left(\left(1-\phi_{0}\right) \mathbb{C}_{s}-\mathbb{C}^{d r, \theta}\right): \mathbb{C}_{s}^{-1}: \boldsymbol{\kappa}_{s}
\end{aligned}
$$

If the solid is much stiffer than the porous material, i.e. if $\left|\mathbb{C}_{s}^{-1}: \mathbb{C}^{d r, \theta}\right| \ll 1$, the above relationships reduce to:

$$
\begin{aligned}
\boldsymbol{B} & =\boldsymbol{\delta} \\
\boldsymbol{K} & =\boldsymbol{\kappa}_{s}: \mathbb{C}_{s}^{-1}: \mathbb{C}^{d r, \theta} \\
L_{s} & =-3\left(1-\phi_{0}\right) \alpha_{s} \text { with } \alpha_{s}=\boldsymbol{\kappa}_{s}: \mathbb{C}_{s}^{-1}: \boldsymbol{\delta} / 3 \\
\frac{1}{N} & =\frac{1-\phi_{0}}{k_{s}} \text { with } \frac{1}{k_{s}}=\boldsymbol{\delta}: \mathbb{C}_{s}^{-1}: \boldsymbol{\delta} \\
C_{s} & =\left(1-\phi_{0}\right)\left(c_{s}+\boldsymbol{\kappa}_{s}: \mathbb{C}_{s}^{-1}: \boldsymbol{\kappa}_{s}\right)
\end{aligned}
$$

The assumption $\left|\mathbb{C}_{s}^{-1}: \mathbb{C}^{d r, \theta}\right| \ll 1$ is made in this paper.

7.3.2. Undrained and adiabatic constraints The state equations of the fluid relate the density $\rho_{f}$ and the mass density of entropy $s_{m, f}$ to pressure and temperature:

$$
\begin{aligned}
& \frac{1}{\rho_{f}}=\frac{1}{\rho_{f, 0}}-\frac{p-p_{0}}{\rho_{f, 0} k_{f}}+\frac{3 \alpha_{f}}{\rho_{f, 0}} \theta \\
& s_{m, f}=s_{m, f, 0}-\frac{3 \alpha_{f}}{\rho_{f, 0}}\left(p-p_{0}\right)+c_{m, f} \theta
\end{aligned}
$$

In undrained conditions, the mass of the fluid in the pore space is a constant, that is $\rho_{f} \phi=\rho_{f, 0} \phi_{0}$, which also reads:

$$
\frac{\phi-\phi_{0}}{\rho_{f, 0}}=\phi_{0}\left(\frac{1}{\rho_{f}}-\frac{1}{\rho_{f, 0}}\right)
$$

Combining (62) and (65), one obtains the undrained constraint between strain, pressure and temperature:

$$
0=\boldsymbol{B}: \boldsymbol{E}+\frac{p-p_{0}}{M}+L \theta
$$


where $\boldsymbol{B}=\boldsymbol{\delta}, L=-3 \bar{\alpha}$ and $M^{-1}=\overline{k^{-1}}$ if $\left|\mathbb{C}_{s}^{-1}: \mathbb{C}^{d r, \theta}\right| \ll 1$.

In undrained conditions, the volume density $S_{f}$ of entropy of the fluid at the macroscopic scale derived from $S_{f}=\rho_{f, 0} \phi_{0} s_{m}$, and (65) reads:

$$
S_{f}=S_{f, 0}-3 \alpha_{f} \phi_{0}\left(p-p_{0}\right)+C_{f} \theta
$$

where $C_{f}=c_{f} \rho_{f, 0} \phi_{0}$. The volume density $S=S_{s}+S_{f}$ of entropy of the porous medium therefore reads:

$$
S=S_{0}+\boldsymbol{K}: \boldsymbol{E}+L\left(p-p_{0}\right)+C \theta
$$

where $C=\bar{c}+\left(1-\phi_{0}\right) \boldsymbol{\kappa}_{s}: \mathbb{C}_{s}^{-1}: \boldsymbol{\kappa}_{s}$ if $\left|\mathbb{C}_{s}^{-1}: \mathbb{C}^{d r, \theta}\right| \ll 1$.

In a reversible adiabatic evolution of the porous material, the entropy is constant. The following adiabatic constraint between strain, pressure and temperature therefore holds in undrained conditions:

$$
0=\boldsymbol{K}: \boldsymbol{E}+L\left(p-p_{0}\right)+C \theta
$$

Eventually, in a reversible undrained adiabatic evolution of the porous medium, it is possible to derive the pore pressure and temperature changes as functions of the strain tensor $\boldsymbol{E}$. It suffices to solve the system of constraints (66) and (69) with respect to $p-p_{0}$ and $\theta$. Introducing the result into the first equation of (62) yields a formally standard linear relationship $\Sigma=\mathbb{C}^{u, a d}: \boldsymbol{E}$ between strain and stress macroscopic tensors, in which the undrained adiabatic stiffness tensor reads:

$$
\mathbb{C}^{u, a d}=\mathbb{C}^{d r, \theta}+M \boldsymbol{B} \otimes \boldsymbol{B}+\frac{1}{C-M L^{2}}(\boldsymbol{K}-M L \boldsymbol{B}) \otimes(\boldsymbol{K}-M L \boldsymbol{B})
$$

From an energy point of view, a very useful property of $\mathbb{C}^{u, a d}$ is that it provides a simple expression of the overall internal energy of the porous material (solid and fluid) in the form:

$$
U=U_{0}+\boldsymbol{\Sigma}_{0}: \boldsymbol{E}+\frac{1}{2} \boldsymbol{E}: \mathbb{C}^{u, a d}: \boldsymbol{E}
$$

\subsection{Fundamentals of the thermodynamics of physical state change}

Let $s_{\ell}(T)$ (resp. $s_{v}(T)$ ) denote the entropy of the unit mass of liquid (rep. vapour). These quantities are related to the enthalpy of vaporization $\Delta H_{\ell \rightarrow v}(T)$ :

$$
T\left(s_{v}(T)-s_{\ell}(T)\right)=\Delta H_{\ell \rightarrow v}(T)
$$

The liquid being a condensed phase, the entropy change is controled by the temperature change:

$$
s_{\ell}\left(T_{2}\right)-s_{\ell}\left(T_{1}\right)=C_{\ell} \log \frac{T_{2}}{T_{1}}
$$

where $C_{\ell}$ is the thermal capacity of the unit mass of liquid. The conservation of fluid entropy in the reversible adiabatic partial vaporization (53) then also reads:

$$
C_{\ell} \log \frac{T^{*}}{T_{0}}+\left(1-x_{\ell}\right) \frac{\Delta H_{\ell \rightarrow v}\left(T^{*}\right)}{T^{*}}=0
$$

Let $v_{v}$ denote the volume of the unit mass of vapour. In the case of a liquid-vapour equilibrium, the gaz pressure is equal to the saturation vapour pressure $p_{v}^{s a t}(T)$ and $v_{v}$ then only depends on temperature and can be related to $p_{v}^{\text {sat }}(T)$ by the perfect gaz law:

$$
v_{v}=\frac{R T}{M_{m} p_{v}^{\text {sat }}(T)}
$$

where $M_{m}$ is the molar mass of the fluid.

For water, the saturation vapour pressure $p_{v}^{s a t}(T)$ can be approximated by the Rankine formula (pressure in bars, temperature in Kelvin):

$$
p_{v}^{\text {sat }}(T)=\exp \left(13.7-\frac{5120}{T}\right)
$$




\section{REFERENCES}

[1] Griffith A. A., The phenomenon of rupture and flow in solids, Philosophical Transactions of the Royal Society of London, vol. 221, 163-198, 1921.

[2] Sneddon I. N. and Lowengrub M., Crack problems in the classical theory of elasticity, Wiley, 1969.

[3] Dormieux, L. and Kondo, D., Micromechanics of fracture and damage, ISTE-Wiley, 2016.

[4] Dormieux, L., Kondo, D. and Ulm F.-J. , Microporomechanics, Wiley, 2006.

[5] Pham, K and Marigo, J.-J., Gradient damage models and their use to approximate brittle fracture, International Journal of Damage Mechanics, vol. 20, 4, 618-652, 2011.

[6] Pham, K and Marigo, J.-J., From the onset of damage to rupture: construction of responses with damage localization for a general class of gradient damage models, Continuum Mechanics and Thermodynamics, vol. 25, 2, 147-171, 2013.

[7] Marigo, J.-J.,Constitutive relations in Plasticity, Damage and Fracture Mechanics based on a work property, Nuclear Eng. Design, 114, 249-272, 1989.

[8] Rice J.R., Thermodynamics of the quasi-static growth of Griffith cracks, Journal of the Mechanics and Physics of Solids, vol. 26, 61-78, 1978.

[9] Sih G. S., Handbook of stress intensity factors, Lehigh University, Bethlehem, 1973

[10] Kachanov M., Elastic solids with many cracks related problems, Academic Press, San Diego, 1993.

[11] Budiansky B. and O'Connell R., Elastic moduli of a cracked solid, International Journal of Solids and Structures, vol.12, 81-97, 1976.

[12] Horii H. and Nemat-Nasser S., Overall elastic modulii with microcracks: load-induced anisotropy, Journal of the Mechanics and Physics of Solids, vol. 31, 155-171, 1983

[13] Ponte-Castaneda P. and Willis J.R., The effect of spatial distribution on the effective behavior of composite materials and cracked media, Journal of the Mechanics and Physics of Solids, vol. 43, 12, 1919-1951, 1995.

[14] Leguillon D., Strength or toughness: a criterion for crack onset at a notch. European Journal of Mechanics - A/Solids, vol. 21, 61-72, 2002.

[15] Carlioz T., Nucléation et propagation de fissures en conditions anisotropes, Thèse de doctorat, Université Paris-Est, 2017.

[16] Coussy O., Mechanics and Physics of Porous Solids. Wiley, 2010.

[17] Hill R., A general theory of uniqueness and stability in elastic-plastic solids, Journal of the Mechanics and Physics of Solids, vol. 6, 236-249, 1958. 